\title{
Cluster observations of the substructure of a flux transfer event: analysis of high-time-resolution particle data
}

\author{
A. Varsani ${ }^{1}$, C. J. Owen ${ }^{1}$, A. N. Fazakerley ${ }^{1}$, C. Forsyth ${ }^{1}$, A. P. Walsh ${ }^{1, *}$, M. André ${ }^{2}$, I. Dandouras ${ }^{3}$, and C. M. Carr ${ }^{4}$ \\ ${ }^{1}$ UCL Mullard Space Science Laboratory, Holmbury St. Mary, Dorking, RH5 6NT, UK \\ ${ }^{2}$ Swedish Institute of Space Physics, Uppsala, Sweden \\ ${ }^{3}$ IRAP, CNRS/Université de Toulouse, Toulouse, France \\ ${ }^{4}$ Blackett Laboratory, Imperial College, London, UK \\ *now at: Science and Robotic Exploration Directorate, European Space Agency, ESAC, Villanueva de la Cañada, \\ Madrid, Spain
}

Correspondence to: A. Varsani (a.varsani.11@ucl.ac.uk)

Received: 14 May 2014 - Revised: 30 July 2014 - Accepted: 30 July 2014 - Published: 8 September 2014

\begin{abstract}
Flux transfer events (FTEs) are signatures of transient reconnection at the dayside magnetopause, transporting flux from the dayside of the magnetosphere into the magnetotail lobes. They have previously been observed to contain a combination of magnetosheath and magnetospheric plasma. On 12 February 2007, the four Cluster spacecraft were widely separated across the magnetopause and observed a crater-like FTE as they crossed the Earth's dayside magnetopause through its low-latitude boundary layer. The particle instruments on the Cluster spacecraft were in burst mode and returning data providing 3-D velocity distribution functions (VDFs) at $4 \mathrm{~s}$ resolution during the observation of this FTE. Moreover, the magnetic field observed during the event remained closely aligned with the spacecraft spin axis and thus we have been able to use these 3-D data to reconstruct nearly full pitch angle distributions of electrons and ions at high time resolution (up to 32 times faster than available from the normal mode data stream). These observations within the boundary layer and inside the core of the FTE show that both the interior and the surrounding structure of the FTE consist of multiple individual layers of plasma, in greater number than previously identified. Our observations show a cold plasma inside the core, a thin layer of antiparallel-moving electrons at the edge of FTE itself, and field-aligned ions with Alfvénic speeds at the trailing edge of the FTE. We discuss the plasma characteristics in these FTE layers, their possible relevance to the magnetopause reconnection processes and attempt to distinguish which of the various different FTE models may be relevant in this case. These
\end{abstract}

data are particularly relevant given the impending launch of NASA's MMS mission, for which similar observations are expected to be more routine.

Keywords. Magnetospheric physics (magnetopause, cusp, and boundary layers)

\section{Introduction}

Half a century has passed since the general relevance of the magnetic reconnection process to the terrestrial magnetosphere was proposed for the first time by Dungey (1961). It is now widely accepted that reconnection is the primary mechanism responsible for coupling mass and energy of the solar wind into the Earth's magnetosphere. Paschmann et al. (1979) were the first to report observations of accelerated flows at the magnetopause boundary layer. Sonnerup et al. (1981) later demonstrated the applicability of the magnetic field and plasma stress balance conditions for reconnected field lines at this boundary. These results were accepted as strong circumstantial evidence that magnetic reconnection occurs at the magnetopause.

Russell and Elphic (1978) reported that spacecraft crossing the magnetopause often observed a bipolar signature in the magnetic field component normal to the magnetopause, which they termed a flux transfer event (FTE). This signature may be associated with an enhancement in the magnetic field intensity or a "crater" in the magnetic field strength (e.g. Paschmann et al., 1982; LaBelle et al., 1987; Farrugia et al., 
1988, 2011; Owen et al., 2008). Other studies have shown that these signatures are consistent with transient reconnection at the magnetopause. FTE signatures are observed at the dayside magnetopause, predominantly when the interplanetary magnetic field (IMF) has a southward component (Rijnbeek et al., 1984; Berchem and Russell, 1984). During northward IMF, reconnection occurs at higher latitudes, in which case FTEs could be observed at the post-terminator magnetopause (Kawano and Russell, 1997; Fear et al., 2005, 2008).

The properties and structure of FTEs have been the subject of many studies over the last two decades. Such studies (e.g. Daly et al., 1984; Paschmann et al., 1982; Rijnbeek et al., 1982, 1984; Sibeck and Siscoe, 1984; Saunders et al., 1984; Scholer, 1988; Southwood et al., 1988) showed that FTE observations can be broadly separated into two groups: observations in which the spacecraft passed into the reconnected magnetic flux tube itself and observations of the magnetic field draped around the reconnected flux tube. The observations from within the reconnected flux tube can be distinguished from those of the draped field region by a change in the local plasma properties coincident with the magnetic field signature. However, Rijnbeek et al. (1987) identified a third plasma regime in the boundary of such structures. They suggested that these regions may contain newly opened field lines, while the field lines within the core of FTE would have been reconnected at some point in the past. FTEs may also be seen both inside the magnetosphere or the magnetosheath and contain plasma from either or both regimes (e.g. Thomsen et al., 1987). The distinctive composition of the plasma in an FTE was demonstrated by Klumpar et al. (1990), who concluded that this was evidence of FTEs being associated with ongoing reconnection.

Some researchers (e.g. Sibeck, 1990, 1992; Sibeck and Smith, 1992) suggested that a transient pressure pulse in the solar wind can generate an FTE-like signature, if the low-latitude boundary layer and magnetopause temporarily pass across the spacecraft location such that the spacecraft briefly enters the plasma depletion layer and magnetosheath. Further studies of particle distributions during "crater" FTE observations showed that this picture was not applicable to all the signatures, and therefore the reconnection mechanism for FTE formation should be retained (e.g. Lockwood, 1991; Smith and Owen, 1992; Elphic et al., 1994; Song et al., 1994). In addition, ionospheric signatures observed during ongoing FTEs at the dayside magnetopause have also been interpreted as support for the reconnection model (e.g. Goertz et al., 1985; Southwood, 1987; Lockwood et al., 1990; Øieroset et al., 1996; Rodger and Pinnock, 1997).

Multi-spacecraft studies have proven to be an invaluable tool (e.g. Russell et al., 1983) to better understand the FTE structure and velocity. Observations by the four-spacecraft Cluster mission (Escoubet et al., 2001) have contributed to analysis of the motion of FTEs and the open magnetic field lines at the magnetopause (e.g. Owen et al., 2001, 2008; Wild et al., 2001; Dunlop et al., 2005; Fear et al., 2005, 2008, 2009, 2010, 2012; Hasegawa et al., 2006, 2010; Farrugia et al., 2011). The tetrahedral configuration of the four spacecraft and their variable separation have provided a platform for reconstruction methods to determine the cross-sectional profiles of FTEs using, for example, Grad-Shafranov techniques (e.g. Sonnerup et al., 2004; Hasegawa et al., 2006). Furthermore, Cluster has opened the window to studying the microphysics of reconnection site (e.g. Fear et al., 2009) and its diffusion region at the magnetopause (André et al., 2004, 2010; Vaivads et al., 2004; Mozer et al., 2005; Retinò et al., 2006; Scudder et al., 2008, 2012). Fear et al. (2008) suggested that different FTE models can be summarized in three types:

i. The elbow-shaped flux-bundle FTEs (Russell and Elphic, 1978), which are postulated to be formed by a short burst of reconnection and occur in pairs which propagate northward and southward away from the reconnection site towards the magnetic poles. These reconnected flux tubes are initially aligned with magnetospheric and magnetosheath magnetic fields on either side of the magnetopause, and are connected, forming the elbow at the reconnection site, providing a route through the magnetopause for plasma to enter and exit. As these flux tubes recede form the reconnection site, the internal magnetic field lines may assume a helical form (Cowley, 1982; Paschmann et al., 1982), while in the immediately exterior regions unreconnected fields may become draped over the structure. Bipolar $B_{N}$ signatures may thus be observed in both the magnetosheath and magnetosphere. When the magnetosheath and magnetosphere magnetic fields are antiparallel (IMF strongly southward), the flux tubes may remain narrow in the azimuthal (dawn-dusk) extent.

ii. Multiple X-line FTEs (Lee and Fu, 1985) can be formed between two (or more) reconnection lines, where magnetosheath and magnetospheric magnetic fields create a helical magnetic field structure that can extend azimuthally over long distances. In this model the bipolar $B_{N}$ signature is observed inside the flux tube or in the draping fields outside. Outside the FTE, open magnetic fields and plasma signatures of reconnection may be observed (Hasegawa et al., 2010).

iii. Single X-line FTEs (Southwood et al., 1988; Scholer, 1988) are formed through a bursty reconnection process. In simple terms, as the reconnection rate increases, the angle between magnetopause plane and open fields become wider (Owen and Cowley, 1987), and a bulge will appear as the thermal pressure increases inside the plasma (Southwood et al., 1988). As in the elbow model, when the FTE moves away from the reconnection site, the bipolar $B_{N}$ signature will be observed both inside the structure and in the draped field lines around 
the FTE. However, as in the Multiple X-line model, it can have a significant azimuthal extent.

Despite the abundance of FTE observations, their formation mechanism is not yet fully understood. In general, higher resolution spatio-temporal measurements are still needed to reveal their detailed structure and to link the observed properties to those of the formation site. We attempt to address this deficiency here by making use of the highest possible timeresolution multi-spacecraft data currently available from the Cluster mission.

In this paper, we present observations from an outbound crossing of the Cluster spacecraft through the low-latitude boundary layer, which occurred on 12 February 2007. On this day, the four spacecraft were deployed in the "multiscale" formation with separations between individual pairs of spacecraft of either $\sim 8000$ or $\sim 800 \mathrm{~km}$. During the interval 10:00 to 10:10 UT, the spacecraft observed bipolar signatures and then made a rapid outbound crossing of the postnoon dayside magnetopause. Solar wind observations from Geotail, ACE and Wind showed a step increase in the dynamic pressure arriving at the dayside magnetopause during the interval of interest. We utilize the high-time-resolution pitch angle distributions of electrons from PEACE instrument along with the high-time-resolution electric (EFW) and magnetic (FGM) field data and ion distributions (CIS) to study these structures in unprecedented detail.

\section{Instrumentation}

In this paper we present magnetopause observations from the four-spacecraft Cluster mission (Escoubet et al., 2001), using data from the Plasma Electron and Current Experiment (PEACE) instruments (Johnstone et al., 1997; Fazakerley et al., 2010), the Cluster Ion Spectrometer (CIS) instruments (Rème et al., 2001), the Cluster Fluxgate Magnetometers (FGM) (Balogh et al., 2001) and the Electric Field and Wave (EFW) instruments (Gustafsson et al., 1997). The upstream solar wind conditions are derived from Geotail (Mukai et al., 1994; Kokubun et al., 1994), Wind (Lepping et al., 1995; Ogilvie et al., 1995) and ACE (Stone et al., 1998) observations.

The four identical PEACE electron spectrometers on Cluster measure the 3-D velocity distribution of electrons in the energy range $\sim 0.7$ to $\sim 27000 \mathrm{eV}$ during a spacecraft spin (four seconds). Each instrument consists of two sensors; the High Energy Electron Analyser (HEEA) and the Low Energy Electron Analyser (LEEA), located on opposite sides of the spacecraft. Each sensor samples $4 \pi$ steradians field of view during one spin of the spacecraft, normally covering a subset of the full energy range of the instrument. Given that the full sky is sampled by each sensor only once per spin, the PEACE instrument flight software is utilized to determine, with reference to the magnetic field unit vector provided by FGM, a pitch angle distribution (PAD) of the electrons which can be transmitted to the ground more frequently that the full 3-D measurements while the spacecraft is in normal mode (NM) of operation. When the burst mode (BM) of operation is enabled, PEACE may also transmit to ground selected 3-D velocity distribution data at spin time resolution, although this may contain data summed across polar angle and/or energy bins in order to reduce the impact on the imposed telemetry rate.

Each of the CIS experiments consists of two ion sensors capable of measuring full 3-D ion distributions from thermal energies up to $\sim 40000 \mathrm{eV}$, once per spin. The two sensors, which are based on the top hat electrostatic analyser design, are named the Composition and Distribution Function analyser (CODIF), which provides the mass per charge composition of ions $\left(\mathrm{H}^{+}, \mathrm{He}^{+}, \mathrm{He}^{++}\right.$and $\left.\mathrm{O}^{+}\right)$, and the Hot Ion Analyser (HIA) that is appropriate for ion beam and solar wind measurements. At the time of the event presented in this paper, both CIS sensors on Cluster 2 and the HIA sensor on Cluster 4 were non-functional. Thus, ion distribution and moments data from HIA instruments are only available from Cluster 1 and Cluster 3 and ion composition from CODIF on Cluster 3 and Cluster 4.

Each Cluster spacecraft carries a magnetometer that is made up of two tri-axial fluxgate sensors located on one of the two solid booms of the spacecraft. During normal mode operations, FGM transmits 22 vectors per second to the ground, while during burst modes 67 vectors per second are transmitted.

The EFW experiment on Cluster is designed to measure the electric-field fluctuations with sampling rates up to 36000 samples a second. The instrument can measure: the quasi-static electric fields of amplitudes up to $700 \mathrm{mV} \mathrm{m}^{-1}$ with high amplitude and time resolution; up to five simultaneous waveforms of a bandwidth of $4 \mathrm{kHz}$ with high time resolution; and density fluctuations at the location of each spacecraft with high time resolution.

\section{Orbit and configuration}

In this paper, we focus on Cluster spacecraft observations made on 12 February 2007 at $\sim 10: 10$ UT, at which time the four spacecraft were located just inside the dayside magnetopause. During the interval of interest, the four spacecraft made an outbound crossing through the magnetopause and its boundary layers. To demonstrate the location of the spacecraft in their orbit, the projection of their positions on the $X Z, X Y$, and $Y Z$ planes are shown in the top row of panels of Fig. 1, in the GSE coordinate system. The projections of the four spacecraft positions on these three planes are represented by the black, red, green and blue circles (for Cluster 1 to 4 respectively). The solid grey curves on each of the panels indicate the intersection of the average bow shock and magnetopause surfaces with each plane. Cluster 3 (C3), which is tracked as the reference spacecraft for this period, was 

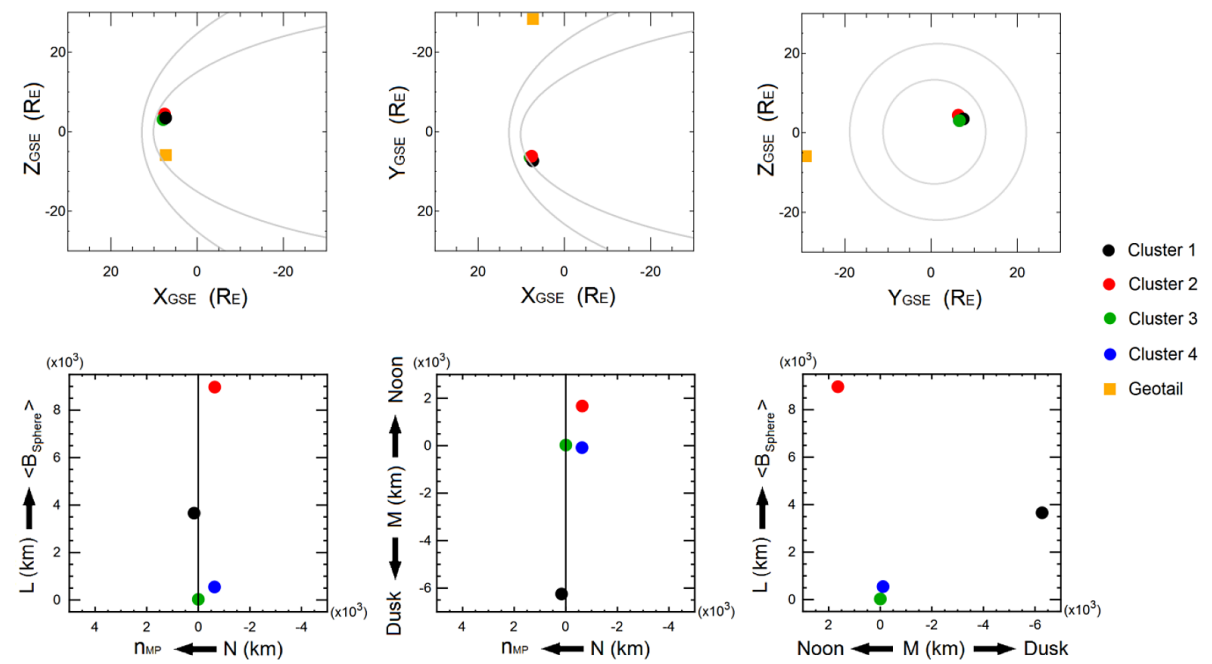

Figure 1. Position of Cluster and Geotail in GSE and LMN coordinates. Top three panels: the location of the Cluster spacecraft at (7.95, $6.55,2.95) R_{\mathrm{E}}$ and Geotail at $(7.24,-29,-5.19) R_{\mathrm{E}}$ at 10:10 UT in (respectively from left to right) the GSE coordinate system $X Z, X Y$ and $Y Z$ planes. The grey lines demonstrate cuts of the model magnetopause based on the solar wind condition (Roelof and Sibeck, 1993, 1994) and bow shock on those planes. Bottom three panels: the configuration of the Cluster tetrahedron in the boundary normal coordinate system derived from the model with $\mathrm{C} 3$ as the reference spacecraft. The grey vertical lines represent the nominal magnetopause boundary. The projections of the four spacecraft locations are shown on the $L N, M N$ and $L M$ planes, from left to right respectively, where $N$ represents the outbound normal to the magnetopause.

located at $(7.95,6.55,2.95) R_{\mathrm{E}}$ in the GSE coordinate system. A fortuitous conjunction with the Geotail spacecraft, located upstream of the magnetopause, occurred during this interval of interest. The location of this spacecraft on the dawn flank at $(7.24,-29,-5.19) R_{\mathrm{E}}$ is represented by the orange square in these plots.

It is useful also to understand the relative locations of the Cluster spacecraft within a natural frame of reference for the examination of magnetopause structures and processes. To achieve this, we determine the orientation of the magnetopause boundary normal coordinate (LMN) system (Russell and Elphic, 1978). We use the Roelof and Sibeck (1993, 1994) magnetopause model to determine the expected local magnetopause normal, $N$, relevant to the spacecraft locations. At the particular position of the reference spacecraft, $N=(0.842,0.489,0.229)$ in the GSE frame, which points predominantly sunward with a minor tilt towards both dusk and the north, consistent with the spacecraft location above the equatorial plane in the post-noon sector. The coordinate $L=(-0.267,0.008,0.964)$ is then taken to lie along the projection of the Earth's magnetic dipole onto the magnetopause while $M=(0.469,-0.872,0.137)$ completes the right-hand set, pointing dawnward in this case.

The relative projections of the four Cluster spacecraft positions on the resultant $L N, M N$ and $L M$ planes are shown in the lower three panels of Fig. 1, using the same colour conventions as the upper panels, and using $\mathrm{C} 3$ as the reference origin. These panels show that $\mathrm{C} 2$ was both closest to the noon meridian and at the highest latitude, while $\mathrm{C} 1$ was located furthest duskward, and C4 was relatively close to $\mathrm{C} 3$, with a separation of $\sim 720 \mathrm{~km}$. Conversely, the relative locations in the $L M$ plane, which is parallel to the expected local magnetopause surface at this location (and is shown in the rightmost part of these panels), indicate the separations of $\mathrm{C} 1, \mathrm{C} 2$ and the $\mathrm{C} 3 / \mathrm{C} 4$ pair are each $\sim 8000 \mathrm{~km}$. Although $\mathrm{C} 1, \mathrm{C} 2$ and $\mathrm{C} 3 / \mathrm{C} 4$ are well separated on the $L M$ plane, the separation in the direction normal to the model boundary is relatively small and comparable to the $\mathrm{C} 3-\mathrm{C} 4$ separation distance. However, if the model boundary is an adequate representation, we expect that, should it move Earthward, C1 should be the first to cross it, then $\mathrm{C} 3$ and finally $\mathrm{C} 2$ and $\mathrm{C} 4$ at roughly at the same time. The consistency of these hypotheses with the observed crossing times is examined in the next section using PEACE and FGM instruments.

\section{Observations}

\subsection{ACE, Wind and Geotail observations}

We have examined data from three different spacecraft, ACE, Wind and Geotail, to determine the solar wind conditions upstream of the Earth, before and during the time of the Cluster observations of interest here. Figure 2 illustrates the data recorded between 09:30 and 10:30 UT by the ACE (red traces), Wind (green traces) and Geotail (blue traces) spacecraft which are located upstream at $(221.5,24.6,11.5) R_{\mathrm{E}}$, $(218.6,-97.7,-16.3) R_{\mathrm{E}}$ and $(7.2,-27.9,-9.6) R_{\mathrm{E}}$ respectively in the GSM system. The data from ACE and Wind are respectively time lagged by 62 and $53 \mathrm{~min}$ to be directly 


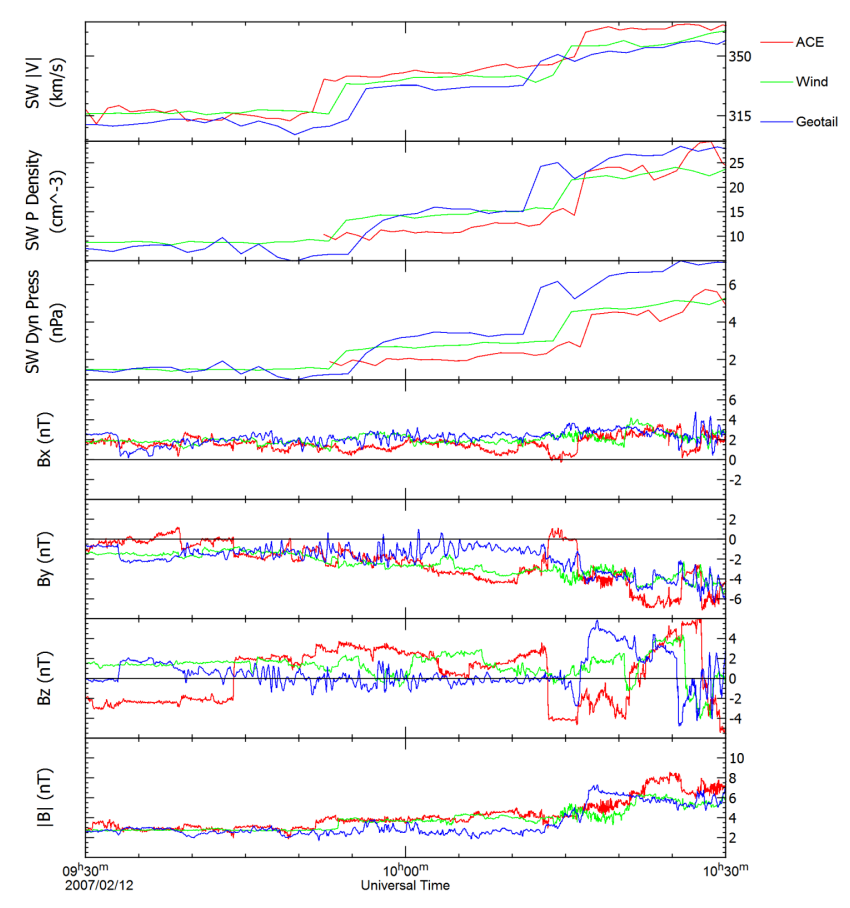

Figure 2. Solar wind and IMF conditions during the time interval 09:30-10:30 UT: solar wind velocity, density and pressure measured at ACE (red traces), Wind (green) and Geotail (blue) respectively are shown on the top three panels. Data from ACE and Wind are respectively lagged by 62 and $53 \mathrm{~min}$ on both plots. These parameters remained steady before 09:30 UT, and then continuously increased over $\sim 3 \mathrm{~h}$ afterwards. The IMF $B_{X}, B_{Y}$ and $B_{Z}$ components in GSM and the magnitude of the field are shown on the bottom four panels; they show multiple orientation variations, while data from Wind suggest the IMF remains northward for most of the time. Geotail recorded $\left\langle B_{Z}\right\rangle \approx 0 \mathrm{nT}$ between 09:40 and 10:15 UT, two significant southward excursions at 10:16 and 10:26 UT, and mostly northward the rest of time. (See Sect. 4.1 for details.)

comparable with the data from Geotail. As the two spacecraft ACE and Wind were separated by only $\sim 2.5 R_{\mathrm{E}}$ along the GSM $X$ direction, the time lag required to align the observations of apparently the same signatures at these two spacecraft suggests that the discontinuity plane in the solar wind flow did not lie fully perpendicular to the Earth-Sun line.

The top three panels of Fig. 2 respectively show the magnitude of velocity, the ion density and the dynamic pressure of the solar wind. The solar wind plasma parameters remained fairly steady for a couple of hours before the interval shown. From the top three panels, it is evident that the increase in the solar wind plasma parameters occurs in two steps, observed by Geotail between 09:56 and 10:11 UT. The time-lagged data from ACE and Wind show a longer time interval between these two steps, which may indicate an ongoing compression of the plasma due to the following faster and denser flow. Unfortunately, density and pressure data before 08:50 UT are not available for ACE (corresponding to the lack of these data prior to 09:52 UT in the time-lagged plots). During this period of time (09:3010:30 UT), the velocity increased in two steps from $\sim 315$ to $\sim 340 \mathrm{~km} \mathrm{~s}^{-1}$ and then $\sim 360 \mathrm{~km} \mathrm{~s}^{-1}$, the density increased from $\sim 5$ to $\sim 15 \mathrm{~cm}^{-3}$ and then to $\sim 25 \mathrm{~cm}^{-3}$ and the pressure increased from $\sim 1.5$ to $\sim 3.5 \mathrm{nPa}$ and then to $\sim 6 \mathrm{nPa}$. As a result of these changes in the solar wind, the Earth's magnetopause is expected to have been compressed Earthward such that, on the basis of models (e.g. Shue et al., 1997; Roelof and Sibeck, 1993, 1994), the sub-solar point standoff distance would have changed from $\sim 10.8$ to $\sim 9.9 R_{\mathrm{E}}$ and then to $\sim 9.2 R_{\mathrm{E}}$. Given the location of the Cluster spacecraft at $(7.95,6.55,2.95) R_{\mathrm{E}}$, we expect these large-scale changes of the solar wind plasma parameters, and consequent magnetopause repositioning, to result in a relative motion of Cluster outbound from the dayside magnetosphere, across the magnetopause and into the magnetosheath.

The bottom four panels in Fig. 2 represent the IMF data in the GSM coordinate system. Although the magnetic field data appear broadly similar in the $6 \mathrm{~h}$ time interval, a closer look at the data in Fig. 2 reveals differences between the observed magnetic fields at each spacecraft. More specifically, the $B_{Z}$ component measured by ACE shows multiple changes in magnetic field orientation between southward and northward at, e.g. 09:43, 10:13, 10:20, 10:27 UT in the lagged data. In contrast, the Wind spacecraft detected a northward IMF for most of the time interval shown, until the IMF switched to southward at 10:27 UT. The data from Geotail show $\left\langle B_{Z}\right\rangle \approx 0$ nT between 09:40 and 10:15 UT, but show two significant southward excursions at 10:16 and 10:26 UT while turning strongly northward between these times. The difference between the magnetic field data observed by the three spacecraft must be related to their wide separation in the $Y$ GSM direction. We note that the Cluster spacecraft and Geotail were also separated by $\sim 34 R_{\mathrm{E}}$ along this direction, which implies some degree of uncertainty to the IMF orientation upstream of Cluster at the time that the solar wind pressure increase reaches the magnetopause. In the next section, we discuss the possible relations between these solar wind conditions and the Cluster observations.

\subsection{Cluster observations: FGM, PEACE and EFW}

An overview of the Cluster observations between 09:52 and 10:12 UT on 12 February 2007 is presented in Fig. 3. The top four sub-panels show the magnetic field components, derived from the data measured by FGM instruments, in the boundary normal (LMN) coordinate system described above. The observations are presented in standard colour code for the Cluster spacecraft, with $\mathrm{C} 1$ data shown as black traces, $\mathrm{C} 2$ in red, C3 in green and C4 in blue. In addition, the lower eight panels in Fig. 3 show the energy spectrograms of electrons that were observed by the PEACE instruments, paired with the total electric field measured by EFW instruments on the four spacecraft, $\mathrm{C} 1$ to $\mathrm{C} 4$ respectively. The energy spectrograms show the direction-averaged differential energy 


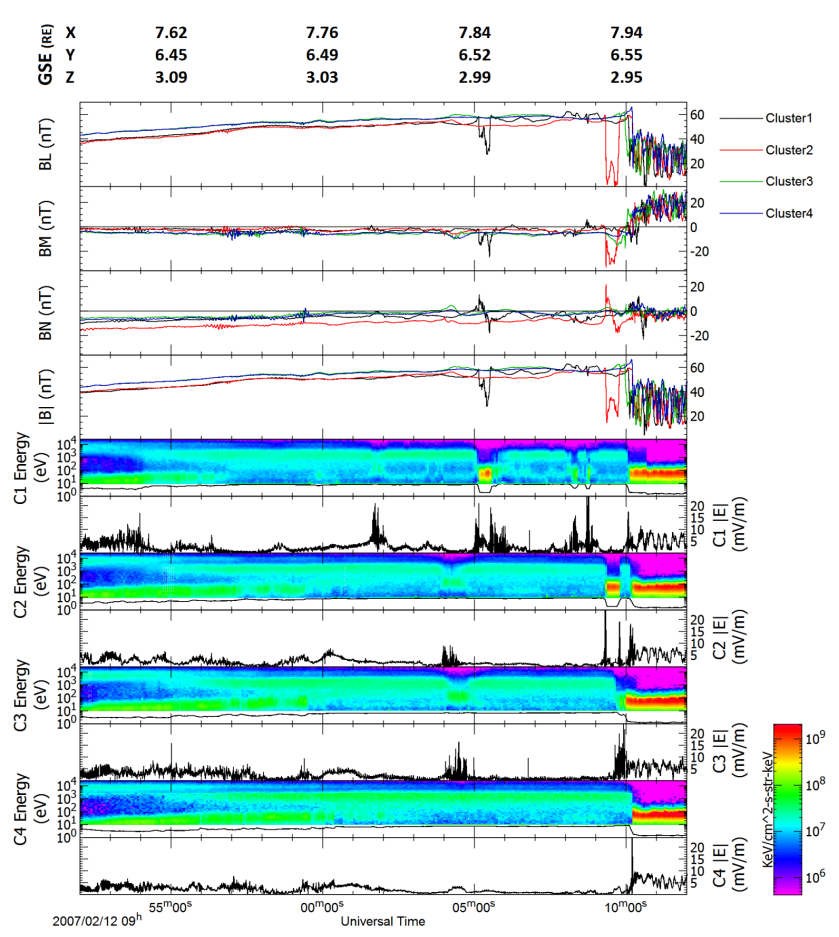

Figure 3. Cluster four-spacecraft observations between 09:52 UT and 10:12 UT: on the top abscissa, the position of reference spacecraft, Cluster 3, is noted for different times. The top four panels show the magnetic field data observed by Cluster FGM in a boundary normal coordinate system, $B_{L}, B_{M}$ and $B_{N}$ components, plus the magnitude of the field, in Cluster standard colour code $(\mathrm{C} 1$ : black, C2: red, C3: green, C4: blue). The bottom four panels show omnidirectional differential energy flux spectrograms from PEACE data (black line represents the spacecraft potential) along with electric field from EFW data on $\mathrm{C} 1, \mathrm{C} 2, \mathrm{C} 3$ and $\mathrm{C} 4$ respectively. The signatures of two separated transient structures consist of a bipolar $B_{N}$ and the appearance of low-energy electron populations are seen before crossing the magnetopause. One is predominately observed by $\mathrm{C} 1$ at 10:05-10:06 UT and the other by $\mathrm{C} 2$ at 10:09-10:10 UT; for the former signature, $\mathrm{C} 2$ and $\mathrm{C} 3$ also observed similar lowenergy electrons between 10:04 and 10:05 UT. All four spacecraft crossed the magnetopause at $\sim 10: 10$ UT. (See Sect. 4.2 for more detail.)

flux of electrons observed at each spacecraft as a function of time (horizontal axis) and electron energy (vertical axes). The coloured pixels represent the flux of the electrons in accordance with the colour bar shown on the extreme right of Fig. 3.

The magnetic field data shown in the top four panels of Fig. 3 indicate a dominant $B_{L}$ component for most of the period prior to $\sim 10: 10 \mathrm{UT}$. During this interval, the $B_{M}$ component remains near zero at all four spacecraft, as expected for a location just inside the dayside magnetopause boundary. The $B_{N}$ component for $\mathrm{C} 1, \mathrm{C} 3$ and $\mathrm{C} 4$ increased from $\sim-15 \mathrm{nT}$ at the start of the period shown to $\sim 0 \mathrm{nT}$ by $\sim$ 10:00 UT. The values of this component have an offset of $\sim-7 \mathrm{nT}$ for $\mathrm{C} 2$, which may be related to the separation of the four spacecraft. At $\sim 10: 10 \mathrm{UT}$, all four Cluster spacecraft observe lower strength and more variable magnetic field. The $B_{L}$ component rapidly falls to around half its earlier value and the $B_{M}$ component increases to $\sim 20 \mathrm{nT}$, consistent with a magnetic field which has both weakened and rotated to be directed northward and dawnward.

The differential energy flux spectrograms for this period, shown in the lower half of Fig. 3 indicate that the spacecraft encounter a number of electron populations during the period shown. Prior to $\sim$ 09:55 UT, each spacecraft detects electrons within two distinguishable energy ranges: (1) a hot population of electrons of higher energy centred on $\sim 6 \mathrm{keV}$; (2) a colder population of electrons with energy centred $\leq 50 \mathrm{eV}$. Between 09:55 and 09:57 UT, the hot population appears to decrease in energy somewhat, becoming centred around $2 \mathrm{keV}$, but largely persists until $\sim 10: 10 \mathrm{UT}$ at each spacecraft. Conversely, the cold population appears to increase in energy, but disappears at each spacecraft before 10:00 UT. At around 10:10 UT, there is an abrupt change in the characteristics of the electron population at each spacecraft. The higher energy populations disappear and are replaced by a lower energy $(\sim 70 \mathrm{eV})$ population at significantly higher fluxes.

Overall, the data for the period shown in Fig. 3 are consistent with the Cluster moving from a location relatively deep inside the magnetosphere towards the dayside magnetopause and out into the magnetosheath. The predominantly strong, northward pointing field and observable fluxes of electrons at high $(>1 \mathrm{keV})$ energy prior to 10:10 UT are consistent with a trapped electron population expected on closed magnetospheric field lines. The lower energy electron population seen in the early part of this period (disappearing before $\sim$ 10:00 UT) may be of ionospheric or plasmaspheric origin, since during this time the spacecraft potential was measured to be below $6 \mathrm{~V}$, indicating these are not likely to be photoelectrons. The abrupt change of both the strength, direction and variability of the magnetic field and the nature of the electron population at all four spacecraft near 10:10 UT indicates that the spacecraft all move from the magnetosphere into the magnetosheath at this time.

Note that there are a number of brief departures evident in Fig. 3 from the overall scenario described above. These are most evident in $\mathrm{C} 1$ data at $\sim$ 10:02, 10:05 and 10:08 UT, but are also seen in C2 and C3 data at $\sim 10: 04$ UT. (Note also that $\mathrm{C} 2$ appears to make a transient $(<1 \mathrm{~min})$ entry into the magnetosheath at $\sim 10: 09 \mathrm{UT}$, returning equally briefly into the magnetosphere at $\sim 10: 10 \mathrm{UT}$ before re-exiting into the magnetosheath and remaining there until the end of the period shown.) The electron differential energy flux spectrograms in Fig. 3 show that $\mathrm{C} 1$ observed low-energy electrons at locations inside the magnetosphere between 10:01-10:02, 10:05-10:06 and 10:08-10:09 UT before crossing the magnetopause at 10:10:04 UT. C2 detected similar electron populations between 10:04-10:05 UT and a denser population between 10:09-10:10 UT, before crossing the magnetopause 
at 10:10:12 UT. C3 also observed similar electrons as C2 between 10:04-10:05 UT and made an outbound crossing at 10:09:58 UT. During the same period of time, despite its proximity to $\mathrm{C} 3, \mathrm{C} 4$ detected only a small variation in high-energy electrons between 10:04-10:05 UT, with no lower energy population, and crossed the magnetopause at 10:10:12 UT.

From the magnetic field data, it is evident that some of these electron signatures were associated with magnetic variations that are consistent with the previous reports of FTElike signatures. In particular, between 10:05 and 10:06 UT, C1 recorded a clear bipolar north-then-south variation in $B_{N}$ component associated with a reduction in the strength of the $B_{L}$ component (and thus $|B|$ ). Moreover, the relative recovery of the strength of this component in the centre of the signature is characteristic of "crater" FTE signatures (LaBelle et al., 1987; Owen et al., 2008; Farrugia et al., 2011). This signature is also associated with two brief negative excursions of the $B_{M}$ component. We also note that the signature is associated with significant enhancements in the electric field wave activity above the levels observed in the magnetosphere outside of the event and later once the spacecraft are located in the magnetosheath.

The similarity of signatures in the electron and electric field data, together with relative timing of other events in the data set shown in Fig. 3 suggest that the signatures observed by C2 and C3 between 10:04 and 10:05 UT may be related to the 10:05-10:06 UT event at C1. All three spacecraft observed a dropout of the high-energy electron fluxes and the appearance of a lower-energy population (albeit to lower flux levels at C2 and C3) in association with enhanced levels of electric field wave activity. The magnetic field signature at $\mathrm{C} 2$ and $\mathrm{C} 3$ was not as prominent as at $\mathrm{C} 1$, but showed mild variation $\left(\Delta B_{N} \lesssim 8 \mathrm{nT}\right)$, which was less than half that observed at $\mathrm{C} 1\left(\Delta B_{N} \sim 20 \mathrm{nT}\right)$. However, neither of these $B_{N}$ signatures were accompanied by a significant change in the magnetic field strength (variance $\lesssim 5 \mathrm{nT}$ ). Assuming that the signatures described above at each of the three spacecraft are of the same event, then the simultaneity of the signatures at $\mathrm{C} 2$ and $\mathrm{C} 3$, together with the relative positions in the LMN coordinate system shown in Fig. 1b, suggests the event has a structure which was elongated and aligned along the $L$ direction, while the delay in the signature at $\mathrm{C} 1$ suggest that the structure was moving duskward.

We note that $\mathrm{C} 1$ observed similar electron and $\boldsymbol{E}$ field signatures to the 10:04-10:05 UT signatures at C2 and C3 at 10:01:30-10:02:00, 10:08:10-10:08:20 and 10:08:4010:08:55 UT. In each case there is almost no significant magnetic field signature associated with these events at $\mathrm{C} 1$, and neither is there any obviously associated signature of any kind at any of the other spacecraft. In addition, we note that $\mathrm{C} 1$ detected a second bipolar change in $B_{N}$ component between 10:10:15 and 10:10:45 UT, immediately after entering the magnetosheath, but without any significant change in other components.
A transient signature was detected by $\mathrm{C} 2$ between 10:09:15 and 10:09:45 UT, just before this spacecraft entered the magnetosheath. The magnetic field variations for this event were very similar to the 10:05-10:06 UT event at $\mathrm{C} 1$, although at somewhat larger magnitudes. However, the electric field variations were confined to the very edges of this event and the electron population was identical to that observed subsequently in the magnetosheath. Although it is tempting to conclude that this may be a transient entry of this spacecraft into the magnetosheath, we note that the deflection of the $B_{M}$ component during this event was negative, i.e. towards dusk, whilst the $B_{M}$ component was observed to be positive (i.e. deflected towards dawn) some $20 \mathrm{~s}$ later when the spacecraft entered the magnetosheath. Also, as we mentioned above, $\mathrm{C} 1$ also observed a bipolar $B_{N}$ signature in the magnetosheath about $1 \mathrm{~min}$ later. This delay is consistent with the similar delay in the variations seen at $\mathrm{C} 2$ and $\mathrm{C} 1$ for the 10:04-10:05 UT event.

Finally, we note that the EFW data from each spacecraft show that for most of the time inside the magnetosphere the electric field had a value of $|E| \lesssim 5 \mathrm{mV} \mathrm{m}^{-1}$, with minimum levels of electric field activity. However, whenever a low-energy electron population was observed by a PEACE instrument, enhanced electric field activity with a level of $\gtrsim 10 \mathrm{mV} \mathrm{m}^{-1}$ was observed at the relevant spacecraft at the same time. This includes the 10:01-10:02, 10:05-10:06 and 10:08-10:09 UT observations by $\mathrm{C} 1$, the 10:04-10:05 and 10:09-10:10 UT by C2 and the 10:04-10:05 UT by C3. Similar activity in the electric field was also seen at the magnetopause boundary for all four spacecraft.

In the remainder of this paper we will concentrate particularly on the details of the signatures of the events observed at C1 at 10:05-10:06 UT, C2, C3 and C4 at 10:04-10:05 UT and their possible relevance. In order to obtain the approximate relative durations of signature passage, we determined the duration of energetic (above $6 \mathrm{keV}$ ) electron dropout observed by the HEEA instrument on each of the four spacecraft: $\mathrm{C} 2 \sim 74 \mathrm{~s}$ from 10:03:41 to 10:04:55 UT, C3 $\sim 85 \mathrm{~s}$ from 10:03:54 to 10:05:19 UT, C4 $\sim 72$ s from 10:04:02 to 10:05:14 UT and C1 88 s from 10:04:51 to 10:06:19 UT. In the next section we present detailed analyses to validate our assumptions on the orientation of the magnetopause boundary local to the Cluster spacecraft at the time of these events.

\subsection{Magnetopause boundary observations}

Initially we attempt to verify our model-based determination of the orientation of the magnetopause boundary described in Sect. 3. We thus employ four-spacecraft timing analysis (Russell et al., 1983; Harvey, 1998; Schwartz et al., 1998) and Minimum Variance Analysis on magnetic field (MVAB) for each spacecraft (Sonnerup and Cahill, 1967; Sonnerup and Scheible, 1998). Figure 4 shows the projection, onto the $L N$ and $M N$ planes, of the four spacecraft positions in standard colour code filled circles with the relative location of 

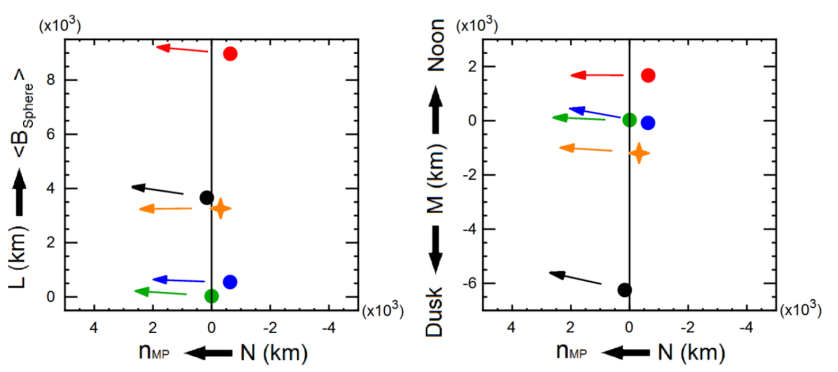

Figure 4. Motion of the magnetopause boundary: the projection of Cluster spacecraft positions onto the $L N$ and $M N$ planes, in standard code coloured filled circles (as in Fig. 1), for the time just before crossing the magnetopause. The model magnetopause boundary is shown as vertical lines. For each spacecraft there is an arrow representing the direction of normal vector calculated using the MVAB method; the four-spacecraft timing analysis is shown as orange arrows, where the four-spacecraft timing analysis velocity is $(0.53,-2.88,-43.82)$ in LMN. (See Sect. 4.3 for details.)

modelled magnetopause at the time of the C3 magnetopause crossing shown as vertical black lines.

The result of MVAB analysis on FGM full-resolution data for the period containing the magnetopause crossing of each spacecraft is represented by the standard code coloured arrows. These are plotted on the assumption that the boundary normal is parallel to the eigenvector associated with the minimum variance direction at each spacecraft. The calculations return an estimate for the magnetopause normal vector $\boldsymbol{N}$ in the reference LMN coordinate system, together with the ratio of intermediate-to-minimum variance eigenvalues $\left(\lambda_{\text {int }} / \lambda_{\min }\right)$ for each spacecraft. These are respectively C1: $N=(0.151$, $0.225,0.962)$ and $\lambda_{\text {int }} / \lambda_{\min } \sim 7.2 ; \mathrm{C} 2: N=(0.094,0.005$, $0.995)$ and $\lambda_{\text {int }} / \lambda_{\min } \sim 1.8 ; \mathrm{C} 3: N=(0.072,0.048,0.996)$ and $\lambda_{\text {int }} / \lambda_{\min } \sim 23$; C4: $N=(0.039,0.189,0.981)$ and $\lambda_{\text {int }} / \lambda_{\min } \sim 127$. Standard practice suggests that the result for $\mathrm{C} 2$ is not well defined given $\lambda_{\text {int }} / \lambda_{\min } \sim 1.8$ and, although generally consistent with the others, this result should be considered with caution.

A further estimate of the boundary normal, together with its velocity, can be obtained using the four-spacecraft timing analysis technique, assuming that the boundary is essentially planar between the locations of the spacecraft. The boundary normal vector calculated using this method is $N=(-0.012$, $0.066,0.998)$ which is represented by the orange arrows emanating from the estimated position of the Cluster barycentre (orange star symbol) in Fig. 4. This method also suggests that the magnetopause was moving Earthward with a velocity in the LMN system of $(0.53,-2.88,-43.82)$, $|V| \sim 43.92 \mathrm{~km} \mathrm{~s}^{-1}$. We note that this is much faster than the orbital velocity of the Cluster spacecraft which for $\mathrm{C} 3$ was $(-1.53,0.31,1.98),|V| \sim 2.52 \mathrm{~km} \mathrm{~s}^{-1}$ at the time of the magnetopause crossing. From the arrows in Fig. 4, it can be seen that all the data-based derivations of the direction of the magnetopause normal vector are in good agreement with the model result. The angle between model normal vector and MVAB analysis of magnetic field for each spacecraft are $\mathrm{C} 1 \sim 16^{\circ}, \mathrm{C} 2 \sim 5^{\circ}, \mathrm{C} 3 \sim 5^{\circ}$ and $\mathrm{C} 4 \sim 11^{\circ}$. These differences may be due to the wide separation of spacecraft on the $L M$ plane, and also small local variations in the MP orientation. We thus conclude that our choice of LMN coordinate system, determined from the models and used above to represent the local magnetopause surface, is sufficiently accurate in this case.

To understand the motion of the FTE-like signature observed by $\mathrm{C} 1$ between 10:05 and 10:06 UT, we further apply the MVAB technique to the spacecraft FGM data during periods covering the entrance and exit to the event. Again assuming that the returned minimum variance direction can be associated with the normal to the boundaries of the event, we find two outbound normal vectors which are respectively $(0.231,0.523,0.821)$ at 10:05:08 UT with intermediateto-minimum ratio $\lambda_{\text {int }} / \lambda_{\min } \sim 8.2$ and $(-0.083,-0.504$, $0.860)$ at $10: 05: 30 \mathrm{UT}$ with intermediate-to-minimum ratio $\lambda_{\text {int }} / \lambda_{\min } \sim 19.8$. These values suggest that the outbound normal vector rotated $\sim 32.5^{\circ}$ dawnwards at the entrance of Cluster 1 into the structure, and then $\sim 30.4^{\circ}$ duskwards at the exit.

Between 10:09 and 10:10 UT, C2, located at higher latitude in comparison with the position of other three spacecraft, observed another transient and potentially FTE-like signature. Using the MVAB method across the boundaries of this event and the same assumptions as above, we find the normal vectors for the entry to the event are $N=(0.053,0.576,0.816)$ with intermediate-to-minimum ratio $\lambda_{\text {int }} / \lambda_{\min } \sim 125$ and for the exit $(0.104,-0.553,0.827)$ intermediate-to-minimum ratio $\lambda_{\text {int }} / \lambda_{\min } \sim 34$. This time, the outbound normal vector rotated $\sim 32.2$ degrees dawnwards at the entrance of Cluster 2 into the structure, and then $\sim 33.8^{\circ}$ duskwards at the exit. As mentioned in the previous section (Sect. 4.2), about $1 \mathrm{~min}$ after this C2 signature, when all four spacecraft had passed into the magnetosheath, C1 again observed a bipolar $B_{N}$ signature. The delay between these signatures is similar to the delay in the variations seen at C2 and C1 for respectively the C2 10:04-10:05 and C1 10:05-10:06 UT events. However, analysis of this FTElike signature is the subject of another study, and thus, in this paper, we only concentrate on high-time-resolution observations of the signature observed by Cluster 1 at 10:0510:06 UT.

\subsection{High-time-resolution observations}

\subsubsection{High-time-resolution pitch angle data}

In normal telemetry modes, 2-D electron pitch angle distributions from the Cluster PEACE instruments are available only once per spin $(\sim 4 \mathrm{~s})$ while 3 -D velocity distributions (3-D VDFs) for electrons are available even less frequently 
(approximately once every minute). Ion 3-D VDFs are available once per spin. In a burst telemetry mode, 3-D electron VDFs with 6 polar bins and 32 azimuthal bins together with 3-D ion VDFs with 8 polar bins and 16 azimuthal bins can be obtained once per spin. Based on the method used by Khotyaintsev et al. (2006) and Schwartz et al. (2011), when the magnetic field vector is closely aligned with the spacecraft spin axis, as is the case for most of the events presented in this paper, these 3-D velocity distributions are equivalent to 16 (ion) or 32 (electron) 2-D pitch angle distributions, thus effectively increasing the available temporal resolution for these data products to $0.250 \mathrm{~s}$ for the ions and $0.125 \mathrm{~s}$ for the electrons. During the event presented in this paper, the orientation of magnetic field inside the magnetosphere remained closely aligned with the spin axis of the spacecraft. Thus this is one of only a few events in which we are able to use this analysis technique to extract relevant particle measurements at time resolutions which are more closely comparable to those available from the electromagnetic field instruments. In the following sections, we make unprecedented presentations of time series of complete, or nearly complete, pitch angle distributions of both electrons and ions at very high time resolution.

\subsubsection{Observations by Cluster 1}

\section{FGM, EFW and PEACE/LEEA observations}

We now look into the details of the FTE encountered by Cluster 1 between 10:05 and 10:06 UT, using high-timeresolution data from the FGM, EFW and PEACE instruments. These data are available during the interval of interest as $\mathrm{C} 1$ was operating in burst mode. The top panel in Fig. 5 shows the magnetic field components in LMN boundary normal coordinate system (the $L$ component is represented by the red trace, $M$ by green and $N$ by blue) at high time resolution $\left(\sim 67\right.$ vectors $\left.\mathrm{s}^{-1}\right)$ for the $1 \mathrm{~min}$ period 10:05-10:06 UT. The second panel contains the $\boldsymbol{E}$ field vector in the same coordinate system and format, presented at a time resolution of $\sim 450$ vectors $s^{-1}$. In the final panel we present the highest available time resolution electron pitch angle distributions for this event. Taking account of the proximity of the magnetic field vector to the spacecraft spin axes in this case, we are able to compute near-complete pitch angle distributions at a time resolution of $0.125 \mathrm{~s}$. Note that this is a considerable improvement on the usual time resolution for this data product which is usually available only at spin $(\sim 4 \mathrm{~s})$ resolution. The data in this bottom panel are presented in the socalled "Sauvaud" format, in which the electron data are split by energy into 26 horizontal mini-panels. Within each minipanel, spectrograms of the electron differential energy flux for the given energy are plotted as a function of pitch angle (vertical axes) versus time (horizontal axes) with $180^{\circ}$ pitch angle particles appearing at the top of each mini-panel and $0^{\circ}$ pitch angle particles appearing at the bottom. The overall presentation then has a dual purpose - viewed at a distance one can gain an impression of the overall energy-time spectrogram of the electrons at this high time resolution, whilst moving up close it is possible to see the variations in pitch angle for electrons of each individual energy band within the plot. We note that each pitch angle distribution is presented with a 15-degree resolution (calculated from the FGM and PEACE data, and rebinned into 12 polar zones). Thus, whenever the angle between spin axis and magnetic field direction is less than $15^{\circ}$, all 12 zones are measured and a full pitch angle distribution is available. However, when this angle is larger, then some pitch angle zones could be missing as the spacecraft spin, most often zones 1 and 12 which cover the parts of the distributions most parallel and anti-parallel to the magnetic field.

On the basis of the data presented in Fig. 5, we divide the event into a number of regions. We first identify three broad classes of region designated "O" (outer), "E" (edge) and "I" (inner), representing a classification of regions "outside", at the "edge" and "inside" the structure we deem responsible for the signatures, based primarily on the departure of the magnetic field from its undisturbed pre-event background. However, given that we are able to examine the structure in this case with unprecedented high-resolution data, we subdivide each of these broad regions into smaller time periods, labelled $\mathrm{I}_{1}, \mathrm{I}_{2}$, etc., according to their detailed signatures. The boundaries of each of these individual regions are marked by the vertical lines in Fig. 5, with our designation for each region marked at the top of the figure. To complement this presentation, Fig. 6 further presents one or more representative pitch angle distributions from each identified layer in Fig. 5, with the order of observation time running from top left to bottom right. In these plots the horizontal axis represents energy in $\mathrm{eV}$, and the vertical axis represents the pitch angle in degrees. Between $\sim$ 10:05:08 and $\sim$ 10:05:32 UT, $\mathrm{C} 1$ observed a bipolar variation in $B_{N}$ component and a significant decrease in $B_{L}$ component, as is evident from the top panel of Fig. 5. This change in magnetic field was accompanied by the appearance of high electron fluxes $\left(\sim 10^{8}\right.$ to $\left.10^{9} \mathrm{keV}\left(\mathrm{cm}^{2} \mathrm{~s} \mathrm{sr} \mathrm{keV}\right)^{-1}\right)$ at low energies, which we take as indicating that the spacecraft was inside the structure itself. We designate regions sampled between the maximum positive $B_{N}$ excursion and the maximum negative $B_{N}$ excursion with the letter "I" in Fig. 5. The two briefly sampled regions in which the magnetic field appears to be varying quasi-monotonically between the exterior background values and the peaks in $B_{N}$ component are deemed to be the edges of the event and are designated as "E". Plasma regions observed before or after these edge regions (i.e. before 10:05:08 and after 10:05:32 UT), where the field is relatively undisturbed, are deemed to be outside the structure and are marked by "O".

Outside the structure, there are time-varying observations of two distinguishable populations of electrons: (i) those in a relatively wide band of energy $(30 \mathrm{eV}$ to $\sim 3 \mathrm{keV})$ but 


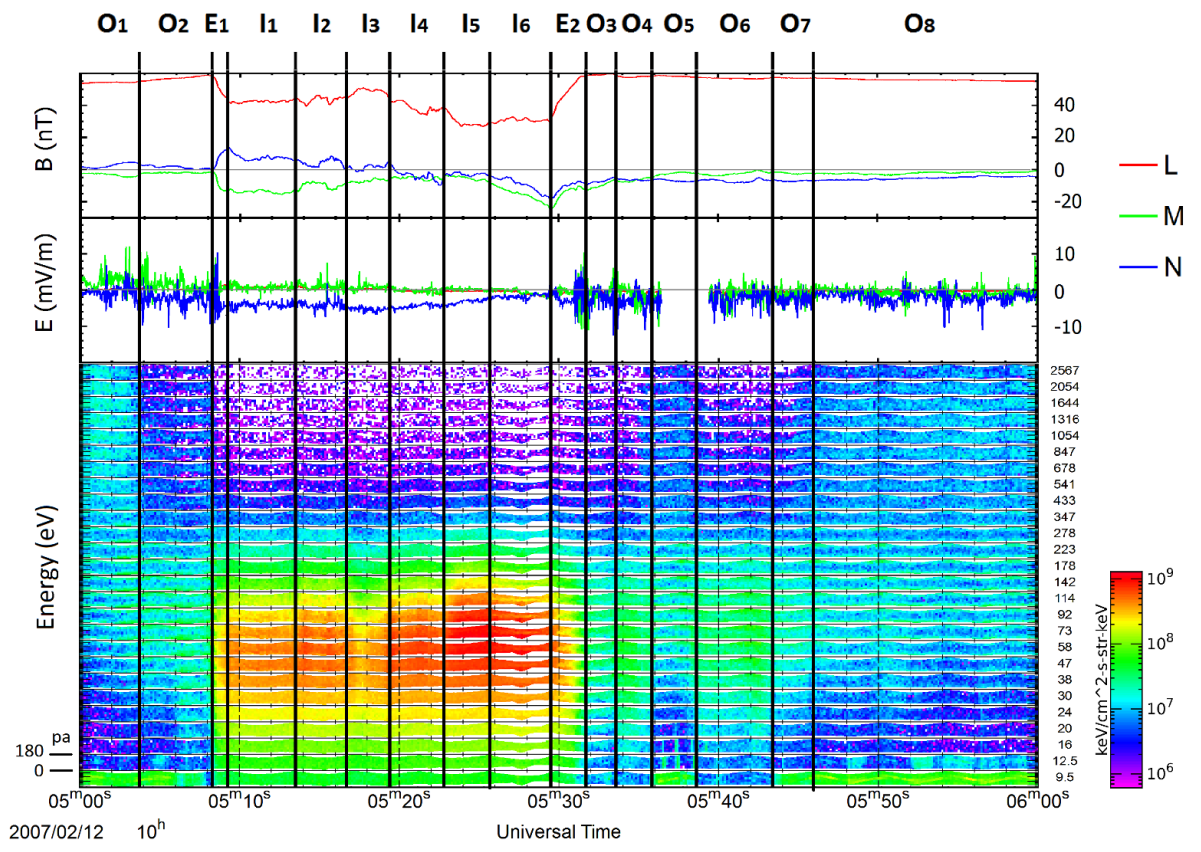

Figure 5. High-time-resolution observations by Cluster 1 from 10:05 UT to 10:06 UT: the top two panels represent magnetic field from FGM instrument and electric field from EFW instrument plotted in the LMN coordinate system, respectively in red, green and blue colour. A horizontal grey line highlights the zero value for each of them. The bottom panel is a "Sauvaud" differential energy flux plot for electron pitch angle distributions from PEACE LEEA instrument, covering energies from $9.5 \mathrm{eV}$ to $2.5 \mathrm{keV}$. The vertical black lines separate different identified regions of the FTE, with their relevant labels on the top. The regions include outer layers marked as "O", edges marked as "E" and inner layers marked as "I". $\mathrm{O}_{1}$ and $\mathrm{O}_{8}$ are the outermost layers of draped magnetic field lines where energetic electrons were observed; $\mathrm{O}_{2}$, $\mathrm{O}_{3}, \mathrm{O}_{5}$ and $\mathrm{O}_{7}$ are inside the boundary layer, with energetic electrons being replaced by bidirectional electrons of $\sim 100 \mathrm{eV}$, and an active electric field; $\mathrm{O}_{4}$ and $\mathrm{O}_{6}$ are still inside the boundary layer, consisting of isotropically distributed electrons with energies $\sim 58 \mathrm{eV}$. $\mathrm{E}_{1}$ is a thin layer of plasma at the edge of the flux tube, where the $B_{L}$ component reduced from its magnetospheric value, the $B_{N}$ component reached its bipolar maximum, the electric field level reached its maximum level and a higher flux of electrons with average energy of $\sim 58 \mathrm{eV}$ developed from the bidirectional electrons of boundary layer. The core labelled as "I" is divided into six layers, where the $B_{N}$ component gradually reached its minimum, the electric field stayed relatively quiet and a general heating up signature is observed in the energy distribution of electrons. Within the inner layer $\mathrm{I}_{3}$, the $B_{L}$ component reached its enhanced level, and a cold low flux population of electrons was observed. $\mathrm{I}_{5}$ had the highest temperature within the core, and inside $\mathrm{I}_{6}$ the electron pitch angle distribution temporarily turned more bidirectional. $\mathrm{E}_{2}$ is the exit edge of FTE. (See Sect. 4.4.2 for details.)

relatively low flux, as seen in regions $\mathrm{O}_{1}, \mathrm{O}_{2}, \mathrm{O}_{3}, \mathrm{O}_{5}, \mathrm{O}_{7}$ and $\mathrm{O}_{8}$, and (ii) those in an energy range $(16 \lesssim E \lesssim 300 \mathrm{eV})$ but with slightly higher flux, as seen in $\mathrm{O}_{4}, \mathrm{O}_{6}$. Note that this energy range is roughly the same as that of the dominant population observed within the structure "I" regions, although that population has significantly higher fluxes. Note also that the presence of lower energy fluxes in regions $\mathrm{O}_{4}, \mathrm{O}_{6}$ is sufficient to rapidly change the value of the spacecraft potential in these regions, as can be deduced from the sudden disappearance of the photoelectron populations in the lowest energy band $(9.5 \mathrm{eV})$ shown in Fig. 5. The observations by $\mathrm{C} 1$ before $\mathrm{O}_{1}$ and after $\mathrm{O}_{8}$ (see Fig. 3) show strong $B_{L}$ component values in magnetic field, $E_{N} \sim 0$ and almost no electric field wave activity and the presence of only the high-energy magnetospheric electrons. These observations suggest that the $\mathrm{O}_{1}$ and $\mathrm{O}_{8}$ regions are sufficiently far from the structure for its perturbing effects to have subsided to the levels of the undisturbed magnetosphere. Indeed, the magnetic field orientation in these two regions was observed to be approximately the same, with strength closest to the level pertaining prior to the observation of the structure.

From Fig. 5, it is clear that during interval $\mathrm{O}_{2}$, the strength of the $B_{L}$ component increased to its maximum value by the end of this interval. This may represent a compression of the field ahead of the approaching structure. The electric field activity observed by the EFW instrument increased in this region and reached its maximum by the end of interval $\mathrm{O}_{2}$. During this interval the PEACE instrument observed both a partial dropout of the higher energy (magnetospheric $\gtrsim 6 \mathrm{keV}$ ) electron population and the first appearance of the population with energies in the lower (magnetosheath $<140 \mathrm{eV}$ ) range. The representative pitch angle distributions from regions $\mathrm{O}_{1}$ and $\mathrm{O}_{2}$ (see Fig. 6, the two leftmost plots in the top row) show that the fluxes of $\mathrm{keV}$ particles disappear around 0 and $180^{\circ}$ pitch angles in the latter 


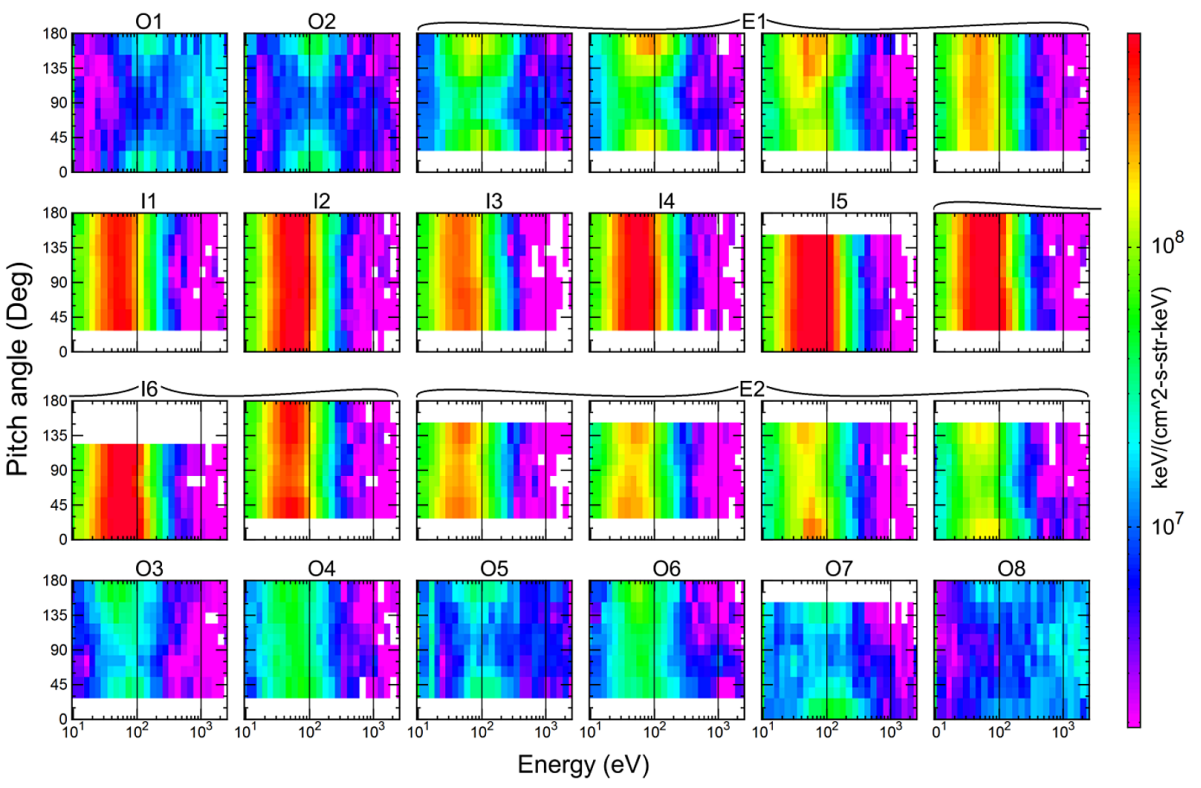

Figure 6. Individual pitch angle distributions from each layer identified in Fig. 5, in the order of observation time from top left to bottom right. The horizontal axis of each plot represents energy in eV, and the vertical axis, pitch angle in degrees. First is $\mathrm{O}_{1}$, where high-energy electrons disappeared around 0 and $180^{\circ}$ pitch angle, while those in the $100 \mathrm{eV}$ range are enhanced at these pitch angles. $\mathrm{O}_{2}$ plot shows higher flux for parallel and anti-parallel electrons in comparison to those which are perpendicular to the magnetic field, with a ratio of fluxes of $\sim 10$. Then there are four consecutive sweeps observed during sub-interval $\mathrm{E}_{1}$, when the initially bidirectional distribution gradually turned into a more isotropic one, broadening first from the antiparallel direction and then spreading into the parallel beam. The next row contains the plots of $\mathrm{I}_{1}$ to $\mathrm{I}_{5}$, which all show a nearly isotropic distribution of electrons, but differ in flux and energy (see Sect. 4.4.2 for details). From $I_{1}$ to $I_{5}$ the peak energy has an increasing trend, except for the central region of FTE, $I_{3}$, which has the lowest flux and density, similar to edges $E_{1}$ and $E_{2}$. Then, three snapshots for region $I_{6}$ are shown, where electrons perpendicular to magnetic field with energies of $\sim 73$ to $\sim 142 \mathrm{eV}$ temporarily disappear, and the distributions become partially bidirectional. Then, there are four panels showing consecutive sweeps of observation in $\mathrm{E}_{2}$, where an isotropic distribution gradually turns bidirectional at the end of this sub-interval. The bottom row of plots contains snapshots outside the FTE, when the core of the FTE has been passed by the spacecraft. In $\mathrm{O}_{3}$ the particles are nearly bidirectional. $\mathrm{In}_{4}$ they become more isotropically distributed. Again in $\mathrm{O}_{5}$ the situation is very similar to $\mathrm{O}_{3}$ which showed clearer bidirectional beams. In $\mathrm{O}_{6}$, the distribution is isotropic, similar to $\mathrm{O}_{4}$, and then again becomes more bidirectional on entering $\mathrm{O}_{7}$. Finally, in $\mathrm{O}_{8}$ electrons show similar behaviour as in $\mathrm{O}_{1}$ as high-energy electrons start to appear. Note that, due to variation of magnetic field orientation, parallel and/or anti-parallel electrons are not measured during certain intervals. (See Sect. 4.4.2 for details.)

region, while those in the $100 \mathrm{eV}$ range are enhanced at these pitch angles.

The first significant change in the magnetic field was detected as the spacecraft entered the region designated as $\mathrm{E}_{1}$. In this region the $B_{L}$ component decreased by $\sim 20 \mathrm{nT}$ from its peak of $\sim 59 \mathrm{nT}$ and the $B_{N}$ component increased from $\sim 0 \mathrm{nT}$ to its maximum $\sim 14 \mathrm{nT}$. During this interval a near-isotropic distribution of electrons with energies between $\sim 30$ and $\sim 73 \mathrm{eV}$ with higher fluxes $\left(>10^{8} \mathrm{keV}\left(\mathrm{cm}^{2} \mathrm{~s} \mathrm{sr} \mathrm{keV}\right)^{-1}\right)$ developed gradually from the more bidirectional distribution observed in region $\mathrm{O}_{2}$. Over this period the perpendicular fluxes of higher-energy electrons also disappeared. Meanwhile, the electric field activity decreased from its maximum at the start of $E_{1}$ to a relatively quiet level at the end of that region. The detailed observation of this development can be seen in the four rightmost panels in the top row of Fig. 6 (labelled by the horizontal bracket marked $\left.\mathrm{E}_{1}\right)$ which cover four consecutive sweeps $(\sim 0.5 \mathrm{~s})$ of the PEACE/LEEA sensor.
Once the spacecraft entered the structure and was located in the regions marked $I_{1}$ to $I_{6}$, we see a general reduction in the $B_{L}$ component values, while the $B_{N}$ component decreases from its maximum of $\sim 15 \mathrm{nT}$ at the beginning of these intervals to its minimum $\sim-18 \mathrm{nT}$ at the end. In addition, the electric field activity remained relatively low, while there were essentially no observations of electrons at magnetospheric energies $(E>0.5 \mathrm{keV})$ and those at lower energies appeared to be distributed rather isotropically through most of the structure interior. More particularly, in region $\mathrm{I}_{1}$, the $B_{L}$ component was roughly steady at $\sim 42 \mathrm{nT}$, the $B_{N}$ component dropped smoothly from 15 to $\sim 6 \mathrm{nT}$, and there was a strong and steady $B_{M}$ component to the field of $\sim-14 \mathrm{nT}$; and isotropic distributions of electrons were observed with peak energies of $\sim 47 \mathrm{eV}$. Inside region $\mathrm{I}_{2}$, the $B_{L}, B_{M}$ and $B_{N}$ component levels were similar to those of $\mathrm{I}_{1}$ although each showed slight variations of order $\pm 3 \mathrm{nT}$, while the electric field showed slightly more activity. However, the fluxes of electrons, while remaining isotropic, increased slightly in 
this region, and the energy of the peak flux also increased to $\sim 58 \mathrm{eV}$, suggesting that an increase in the temperature of the plasma had occurred.

Within region $\mathrm{I}_{3}$, Cluster 1 observed a partial recovery of the $B_{L}$ component of the magnetic field which peaked at an enhanced level of $\sim 52 \mathrm{nT}$ in this interval. The $B_{N}$ component oscillated slightly around a level of $\sim 3 \mathrm{nT}$, while the $B_{M}$ component remained $\sim-6 \mathrm{nT}$. There was a dropout in the fluxes of the electrons in the $30-73 \mathrm{eV}$ energy range, although they remained largely isotropic, as can also be seen in the middle plot of the second row of Fig. 6, labelled $I_{3}$. This electron distribution appears similar to that which developed at the end of region $\mathrm{E}_{1}$ (and is subsequently seen at the beginning of region $\mathrm{E}_{2}$ ). However, although the magnetic field strength also shows something of a recovery, the $B_{N}$ component remains much smaller than is observed at the end of $E_{1}$ (and beginning of $E_{2}$ ) and shows no evidence of any bipolar variation. Moreover, the $\boldsymbol{E}$ field wave activity in this region remains at a very low level.

Within region $\mathrm{I}_{4}$, the $B_{L}$ component was observed again to fall to levels $\sim 38 \mathrm{nT}$, but the $B_{N}$ component became on average negative in this region at $\sim-2 \mathrm{nT}$. The magnitude of the $B_{M}$ component was smallest in this region, with a value $\sim-6 \mathrm{nT}$ (Note that field shows variations up to $\pm 4 \mathrm{nT}$ ); the electric field variations remained at a very low level; the flux of electrons also recovers to levels similar to that seen in region $\mathrm{I}_{2}$ and with a similar energy distribution (i.e. isotropic). As the spacecraft entered region $\mathrm{I}_{5}$, the $B_{L}$ component reduces to $27 \mathrm{nT}$, and $B_{M}$ and $B_{N}$ components to $\sim-6 \mathrm{nT}$. The $\boldsymbol{E}$ field noise levels remain low. The electron population apparently remained near-isotropic. However, it seems clear that the overall electron population had higher fluxes than observed in the preceding regions and that it also had a higher temperature, since the energy of the peak in the differential energy flux rose to $\sim 73 \mathrm{eV}$.

As the spacecraft passed through the final regions designated as "inside", the magnetic field orientation changed significantly. The $B_{L}$ component was relatively steady at the lowest level, $30 \mathrm{nT}$, seen during this entire interval of interest. The $B_{M}$ and $B_{N}$ components became steadily more negative as the spacecraft crossed region $\mathrm{I}_{6}$ and reached their global minima by the end of this period; the $\boldsymbol{E}$ field noise levels remained low; the electron population with energies $\sim 73$ to $\sim 142 \mathrm{eV}$ temporarily changed from near-isotropic to more bidirectional, as the perpendicular electrons disappear in the centre of $\mathrm{I}_{6}$, but again became near-isotropic by the end of the region. This change in pitch angle distribution of electron is shown via three snapshots of region $\mathrm{I}_{6}$ in Fig. 6 . We note that the large change in magnetic field orientation in this region moved it away from the direction of the spacecraft spin axis, thus our ability to sample the full PAD (see Fig. 6, third row) is compromised in this interval, with the loss of observations of the near-field- and anti-field-aligned electrons.

As the spacecraft crossed the region $\mathrm{E}_{2}$, the $B_{L}$ component was observed to increase rapidly and monotonically by $\sim 30 \mathrm{nT}$ to reach a level of $\sim 59 \mathrm{nT}$, very similar to that observed before entering region $\mathrm{E}_{1}$, while the gradient in the $B_{N}$ component reversed from that seen in the interior regions and increased from its minimum of $\sim-18$ to $\sim-8 \mathrm{nT}$. The electric field activity again increased to near the maximum levels observed at the end of this period; the near-isotropic electrons observed at the beginning of the interval $E_{2}$ reduced in flux and became more bidirectional as the spacecraft crossed this region. Although we have incomplete pitch angle coverage in this region, this development is again evident from the four plots of the third row of Fig. 6, which again represent the measurement of four consecutive sweeps by the LEEA sensor over $0.5 \mathrm{~s}$.

Based on the observations of spacecraft in the exterior regions marked $\mathrm{O}_{3}$ to $\mathrm{O}_{7}$ lying in the wake of the structure, the $B_{L}$ component of the magnetic field showed a small gradual decrease to nearer its pre-event level observed in region $\mathrm{O}_{1}$. The $B_{M}$ and $B_{N}$ components remained small but negative and showed only slight variations. The electric field activity was observed to be relatively high throughout this whole post-event region. The electron populations observed in $\mathrm{O}_{3}$, $\mathrm{O}_{5}$ and $\mathrm{O}_{7}$ areas were similar to those in $\mathrm{O}_{2}$, including their energy range, flux and bidirectional distribution. However, the electrons in $\mathrm{O}_{4}$ and $\mathrm{O}_{6}$ were more isotropic, and similar to those observed in the interior regions (marked "I"), but with much lower fluxes. Examples of pitch angle distributions observed in regions $\mathrm{O}_{3}-\mathrm{O}_{7}$ are shown in the bottom row of plots in Fig. 6.

\section{CIS/HIA instrument}

Before attempting to interpret the observations described in the previous section, it is useful to consider what information can also be obtained from the ion instruments on the Cluster spacecraft. In principle, our technique for obtaining hightime-resolution pitch angle slices can also be applied to the ion data. However, unlike the electrons, whose thermal and gyration velocities are very high compared to any drift velocity, the speed of individual ions is likely to be comparable to the drift velocity. For electrons, this situation leads to an expectation of near-gyrotropy in spacecraft observation frame, and thus a representative pitch angle distribution can usually be obtained from any time slice of the data. For the ions, however, we expect there to be significant variations between time slices due to the anisotropies introduced into the distribution by any drift motion. With this caveat in mind, we now also present the high-time-resolution ion distributions from the HIA high-sensitivity instrument on Cluster 1, which consist of $162-\mathrm{D}$ azimuthal slices through phase space per spin. For ease of comparison with the earlier plot, Fig. 7 shows a plot for the identical time period and in the identical format to Fig. 5, except that we have replaced the bottom panel with the "Sauvaud" format energy distribution for ions obtained from CIS data. The regions discussed above in relation to the electron plot in Fig. 5 are marked at the top of Fig. 7. 


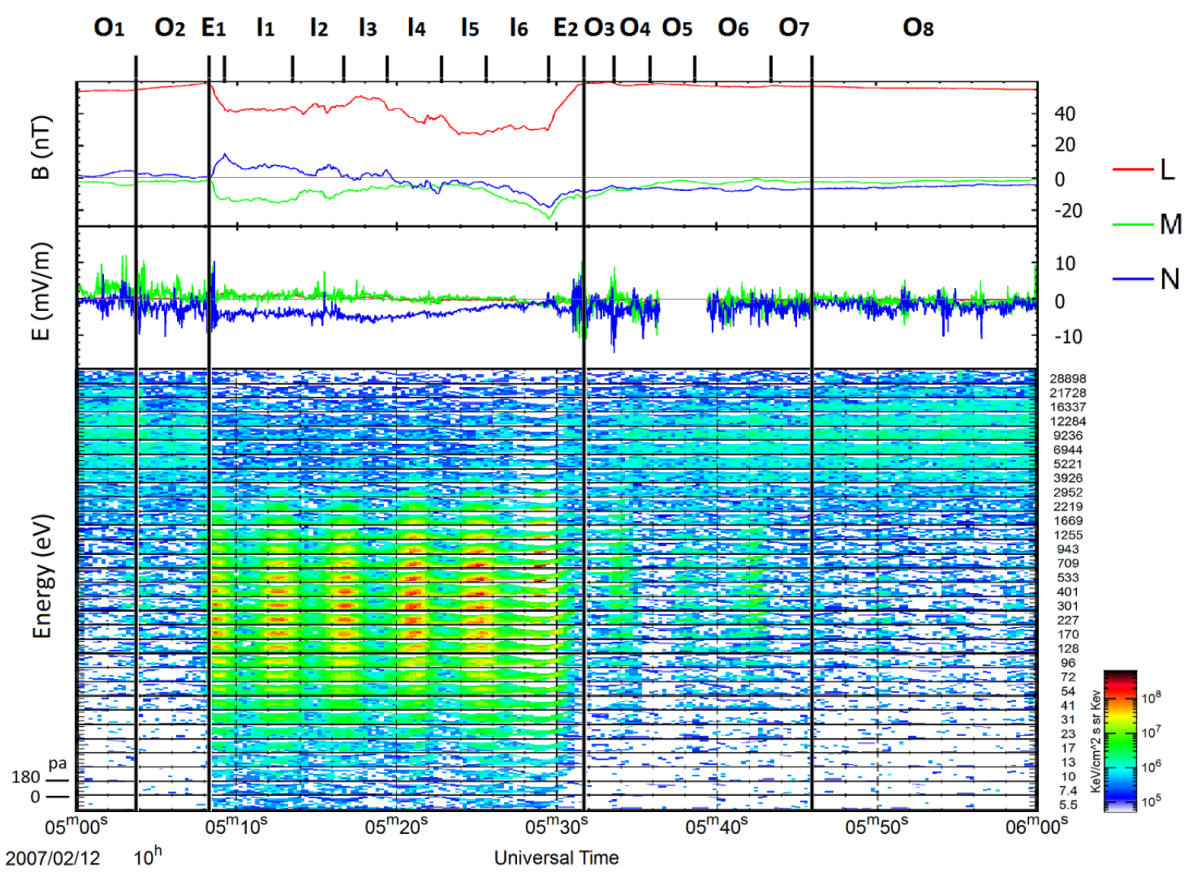

Figure 7. High-time-resolution ion observations from Cluster 1 between 10:05 and 10:06 UT. During this time interval, significant fluxes of high-energy ions were observed above $\sim 4 \mathrm{keV}$ in regions $\mathrm{O}_{1}$ and $\mathrm{O}_{8}$. The ion populations observed in the regions immediately surrounding the structure (in particular the regions labelled $\mathrm{O}_{2}$, and also $\mathrm{O}_{3}-\mathrm{O}_{7}$ ) contained significant fluxes of ions in both the high-energy (similar to those observed in $\mathrm{O}_{1}$ and $\mathrm{O}_{8}$ ) and a lower-energy (between $\sim 50 \mathrm{eV}$ and $2 \mathrm{keV}$ ) bands. From the beginning of region $\mathrm{E}_{1}$ until the end of region $\mathrm{E}_{2}$, the flux of high-energy ions of magnetospheric origin drops, and the main population is centred at low energy between $\sim 50 \mathrm{eV}$ and $2 \mathrm{keV}$. We note that the population with lower energy shows some modulation of flux at close to the spin frequency (4.148 s) in regions $\mathrm{E}_{1}$ to $\mathrm{O}_{7}$. This is a result of the ions with magnetosheath energies not being gyrotropic, and thus the flux of incoming ions increases every time the HIA instrument looks into the flow direction. Inside the structure, there are six points in time at which a representative pitch angle distribution of ions is obtainable. (See Sect.4.4.2 and Fig. 8 for details.)

During the time interval shown in Fig. 7, significant fluxes of high-energy ions were observed above $\sim 4 \mathrm{keV}$ in regions $\mathrm{O}_{1}$ and $\mathrm{O}_{8}$, bracketing the overall event. There are no clear anisotropies, nor clear variation with spin period within these regions. The ion populations observed in the regions immediately surrounding the structure (in particular the region labelled $\mathrm{O}_{2}$, and also $\mathrm{O}_{3}-\mathrm{O}_{7}$ ) contained significant fluxes of ions in both the high-energy (similar to those observed in $\mathrm{O}_{1}$ and $\mathrm{O}_{8}$ ) and a lower-energy (between $\sim 50 \mathrm{eV}$ and $2 \mathrm{keV}$ ) bands. We note that the population with lower energy shows some modulation of flux at close to the spin frequency (4.148 s) in regions $\mathrm{O}_{3}-\mathrm{O}_{7}$.

From the beginning of region $E_{1}$ until the end of region $E_{2}$, the flux of high-energy ions of magnetospheric origin (centred at $\sim 10 \mathrm{keV}$ ) drops, and the main population is predominantly between $\sim 50 \mathrm{eV}$ and $2 \mathrm{keV}$; although there is some evidence of low fluxes which show spin phase modulation at these energies. More importantly, in these edge and interior regions, the lower-energy $(<4 \mathrm{keV})$ ions are observed with relatively very high fluxes. These are again heavily modulated at the spacecraft spin period, indicating that the $\boldsymbol{E} \times \boldsymbol{B}$ drift velocity must be comparable to the ion gyration velocities in these regions. The timing of the flux maxima observed in six consecutive spins every $4.148 \mathrm{~s}$ from 10:05:08.560 to 10:05:28.908 UT suggest that the convection within the structure was in the direction $(-0.19,-0.97,-0.17)$ in the LMN coordinate system. Since there are only six relevant data points for ions, we have calculated the $\boldsymbol{E} \times \boldsymbol{B}$ drift velocity, based on high-time-resolution observations by EFW and FGM instruments on Cluster 1 and assuming $\boldsymbol{E} \cdot \boldsymbol{B}=0$. These velocities, averaged across each region, are presented in Table 1 . The average $\boldsymbol{E} \times \boldsymbol{B}$ drift velocity determined using data from the FGM and EFW instruments for the first five spins during this period was $\sim 101 \mathrm{~km} \mathrm{~s}^{-1}$ in the direction $(-0.18,-0.97,-0.16)$ in $\mathrm{LMN}$, which is consistent with the velocity observed perpendicular to the magnetic field from CIS/HIA instruments. We have ignored the last ion flux peak within this period since at this time the magnetic field orientation made a larger angle to the spin axis of the spacecraft, resulting in reduced accuracy of the $\boldsymbol{E} \times \boldsymbol{B}$ drift velocity estimate due to the lack of knowledge of the spin-axis-aligned electric field component from the EFW instrument field. Within the leading half of the structure (regions $\mathrm{E}_{1}, \mathrm{I}_{1}-\mathrm{I}_{3}$ ) the ions appear to be moving predominantly perpendicular to the magnetic field, while after the enhancement in $B_{L}$ component $\left(\mathrm{I}_{4}, \mathrm{I}_{5}\right.$ and $\left.\mathrm{I}_{6}\right)$ the ion distribution 
Table 1. High-time-resolution $\boldsymbol{E} \times \boldsymbol{B}$ drift velocity in LMN coordinate system, calculated from parameters observed by EFW and FGM instruments on Cluster 1. Note that these values are calculated assuming that $\boldsymbol{E} \cdot \boldsymbol{B}=0$.

\begin{tabular}{llll}
\hline $\begin{array}{l}\text { Observation } \\
\text { region }\end{array}$ & $\begin{array}{l}\text { Velocity } \\
\left(\mathrm{km} \mathrm{s}^{-1}\right)\end{array}$ & $\begin{array}{l}\text { Observation } \\
\text { region }\end{array}$ & $\begin{array}{l}\text { Velocity } \\
\left.\mathrm{km} \mathrm{s}^{-1}\right)\end{array}$ \\
\hline $\mathrm{O}_{1}$ & $(1,-7,-33)$ & $\mathrm{I}_{6}$ & $(-16,-48,24)^{*}$ \\
$\mathrm{O}_{2}$ & $(0,-43,-35)$ & $\mathrm{E}_{2}$ & $(-7,-41,24)$ \\
$\mathrm{E}_{1}$ & $(-7,-60,-19)$ & $\mathrm{O}_{3}$ & $(-3,-35,20)$ \\
$\mathrm{I}_{1}$ & $(-22,-87,-27)$ & $\mathrm{O}_{4}$ & $(-5,-54,11)$ \\
$\mathrm{I}_{2}$ & $(-17,-91,-27)$ & $\mathrm{O}_{5}$ & $(0,-45,24)$ \\
$\mathrm{I}_{3}$ & $(-15,-105,-15)$ & $\mathrm{O}_{6}$ & $(1,-29,13)$ \\
$\mathrm{I}_{4}$ & $(-15,-112,4)$ & $\mathrm{O}_{7}$ & $(1,-43,22)$ \\
$\mathrm{I}_{5}$ & $(-20,-107,-8)$ & $\mathrm{O}_{8}$ & $(0,-13,3)$ \\
* The accuracy of the drift velocity value for region $\mathrm{I}_{6}$ is low, as the magnetic field orientation \\
made a larger angle to the spin axis of the spacecraft.
\end{tabular}

is observed to have components of motion both field-aligned and perpendicular to the field. More specifically, in $\mathrm{I}_{6}$, the ion flow appears to be dominantly in the field-aligned direction. These variations in the ion distribution observed at the time of the peak flux in each spin are illustrated more clearly in the plots presented in Fig. 8. In this figure each panel shows the ion flux averaged over the two sweeps of CIS HIA instrument which bracket the time the sensor looks into the convection direction. We note also a steady increase in the energy band containing the peak flux of the ions observed as the spacecraft crosses the structure, from around $300 \mathrm{eV}$ in region $\mathrm{E}_{1}$ to around $700 \mathrm{eV}$ at the end of region $\mathrm{E}_{2}$.

\subsubsection{Observations by Cluster 2 and 3}

As previously mentioned in Sect. 4.2, in addition to the Cluster 1 observations, Cluster 2 also observed a (relatively minor) bipolar variation in the $B_{N}$ component, along with observations of a low-energy population of electrons, and electric field activity. Figure 9 illustrates the high-timeresolution observations by Cluster 2 between 10:03:45 and 10:04:45 UT, in the same format as Fig. 5. The top two panels represent the magnetic field data from the FGM instrument, and electric field data from the EFW instrument, which are plotted in the LMN coordinate system, respectively as the red, green and blue colours. The bottom panel is a "Sauvaud" energy flux plot for electron pitch angle distribution, from the PEACE LEEA sensor that covers the energies from $12.5 \mathrm{eV}$ to $2.5 \mathrm{keV}$. The magnetic field data from an observation at 10:03:50 UT show that $B_{L}, B_{M}$ and $B_{N}$ values were respectively $\sim 54, \sim-2$ and $\sim-7 \mathrm{nT}$. Observations of EFW and PEACE for the same time show that the electric field had a value of $\sim 2 \mathrm{mV} \mathrm{m}^{-1}$, and the main population of electrons observed was above $1 \mathrm{keV}$. These observations indicate that the spacecraft was completely inside the magnetosphere at that time. However, after $\sim$ 10:03:56 UT, the $B_{N}$ value started to increase, and an increase in the electric field with relatively higher level of activity was observed. In addition to that, electrons with energies above $1 \mathrm{keV}$ started to disappear,
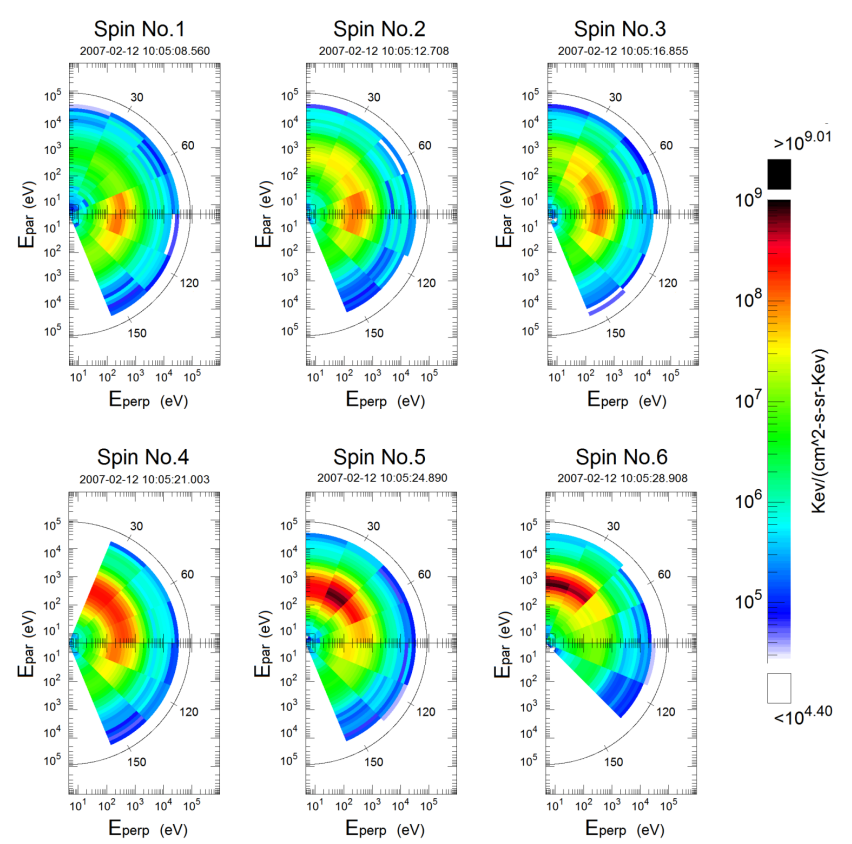

Figure 8. Ion pitch angle distribution in parallel-perpendicular energy space, for six spins of Cluster 1, inside the observed FTE. Each panel shows the ion flux averaged over the two sweeps of CIS HIA instrument which bracket the time the sensor looks in the convection direction. Due to variation in magnetic field orientation, one or two pitch angle zones are missing. In spin No. 1, ions are observed to be moving predominantly perpendicular to the magnetic field ( $\boldsymbol{E} \times \boldsymbol{B}$ drift effect), where the maximum flux has a kinetic energy of $\sim 300 \mathrm{eV}$. In the next two spins, No. 2 and No. 3, the peak energy rises to $\sim 400 \mathrm{eV}$, and has parallel component as well. In spin No. 4, a broader range of ion PAD from 0 to $90^{\circ}$ is observed, along with an increase in the energy band containing the peak flux of the ions, up to $1.2 \mathrm{keV}$. One spin later, in spin No. 5, the ion peak energy increased to $\sim 500-600 \mathrm{eV}$, with a parallel component larger than the perpendicular (identical to pitch angle $33.75^{\circ}$ ). Finally, in the last spin, No. 6 , the ions are observed to be moving mainly parallel to the magnetic field with peak energy of $\sim 700 \mathrm{eV}$.

and instead electrons with energy of $\sim 100 \mathrm{eV}$ were observed. The trend stayed the same, until 10:04:01 UT, when the $B_{N}$ component reached its maximum value of $\sim-5 \mathrm{nT}$, the $B_{L}$ component rose to $\sim 54 \mathrm{nT}$ and the $B_{M}$ component to $\sim-7 \mathrm{nT}$. The electric field increased to $\sim 8 \mathrm{mV} \mathrm{m}^{-1}$, and the main population of electrons had energies of $\sim 100 \mathrm{eV}$, with a bidirectional distribution. After that, the $B_{N}$ component started to decrease to its minimum level $\sim-9 \mathrm{nT}$, at 10:04:08 UT, while the $B_{L}$ component continued to increase and reached its maximum value $\sim 56 \mathrm{nT}$ and the $B_{M}$ component increased to $\sim-3 \mathrm{nT}$. The electric field showed less activity, with values below $\sim 2 \mathrm{mV} \mathrm{m}^{-1}$, but the low-energy population of electrons stayed as before. In other words, between 10:04:01 and 10:04:08 UT, the $B_{N}$ component made a bipolar variation from $\sim-5$ to $\sim-9 \mathrm{nT}$, against a background offset of $\sim-7 \mathrm{nT}$, which is thought to be related to 
the separation of Cluster 2 and Cluster 3 (as the reference spacecraft) in the $L$ direction. Then the $B_{L}, B_{M}$ and $B_{N}$ components respectively reached $\sim 5, \sim-4$ and $\sim-10 \mathrm{nT}$ at 10:04:34 UT, when electrons with energies higher than $1 \mathrm{keV}$ were observed, and the low-energy population slowly disappeared, which indicates that the spacecraft was inside the magnetospheric population of plasma once more. Throughout this period, the distribution of electrons $(\sim 100 \mathrm{eV})$ was mainly bidirectional, and the energy flux of electrons was on average $2 \times 10^{7} \mathrm{keV}\left(\mathrm{cm}^{2} \mathrm{~s} \mathrm{sr} \mathrm{keV}\right)^{-1}$, but fluctuated by a factor of $\sim 2$. No entry to magnetosheath was detected for the whole time interval that this low-energy population was observed.

With a few seconds delay, Cluster 3 recorded a similar sequence of observations. Figure 10 illustrates the high-timeresolution observations by Cluster 3, between 10:03:57 and 10:04:57 UT. The same format of panels used in Figs. 5 and 9 present the data from FGM, EFW and PEACE LEEA. The observations of FGM instrument at 10:04:00 UT, show that the $B_{L}, B_{M}$ and $B_{N}$ values were respectively $\sim 58, \sim-5$ and $\sim 2 \mathrm{nT}$. Observations of EFW and PEACE respectively show that the electric field had a value of $\sim 2 \mathrm{mV} \mathrm{m}^{-1}$, and the main population of electrons was observed above $1 \mathrm{keV}$, which could indicate that the spacecraft was completely inside the magnetosphere. However, after $\sim$ 10:04:04 UT, the $B_{N}$ value started to increase, and an increase in the electric field with relatively higher level of activity was observed. At the same time, electrons with energies above $1 \mathrm{keV}$ started to disappear, and instead electrons with energy of $\sim 100 \mathrm{eV}$ were observed. Then, at 10:04:14 UT, the $B_{N}$ component reached its maximum value of $\sim 5 \mathrm{nT}$, the $B_{L}$ component rose to $\sim 59 \mathrm{nT}$, and the $B_{M}$ component dropped to $\sim-8 \mathrm{nT}$ . The electric field increased to $\sim 5 \mathrm{mV} \mathrm{m}^{-1}$, and the main population of electrons had energies of $\sim 100 \mathrm{eV}$, with a bidirectional distribution. Next, the $B_{N}$ component decreased to $\sim 0 \mathrm{nT}$ at 10:04:27 UT, the $B_{L}$ component reached its maximum $\sim 60.5 \mathrm{nT}$, and the $B_{M}$ component rose to $\sim-7 \mathrm{nT}$. The electric field increased to $\sim 12 \mathrm{mV} \mathrm{m}^{-1}$ with a high level of activity, and $2 \mathrm{~s}$ later reached its global maximum of $\sim 15 \mathrm{mV} \mathrm{m}^{-1}$. Meanwhile, the $B_{N}$ component continued to decrease and reached its minimum level $\sim-4 \mathrm{nT}$, at 10:04:32 UT, while the $B_{L}$ component dropped to $\sim 59.5 \mathrm{nT}$, and the $B_{M}$ component rose to $\sim-4 \mathrm{nT}$. In other words, between 10:04:14 and 10:04:32 UT, the $B_{N}$ component made a bipolar variation from $\sim 5$ to $\sim-4 \mathrm{nT}$. After that, the $B_{L}, B_{M}$ and $B_{N}$ components respectively reached $\sim 59 \mathrm{nT}$, $\sim-5 \mathrm{nT}$ and $\sim-2 \mathrm{nT}$ at 10:04:52 UT, when electrons with energies higher than $1 \mathrm{keV}$ were observed, the low-energy population slowly disappeared and the electric field dropped to $\sim 2 \mathrm{mV} \mathrm{m}^{-1}$ with a low level of activity. This indicates that the spacecraft was inside the undisturbed magnetosphere again. Throughout this period, the distribution of electrons $(\sim 100 \mathrm{eV})$ were mainly bidirectional and the energy flux of electrons was on average $3.5 \times 10^{7} \mathrm{keV}\left(\mathrm{cm}^{2} \mathrm{~s} \mathrm{sr} \mathrm{keV}\right)^{-1}$, with a fluctuation of an order of $\sim 3$. However, no entry to
Table 2. High-time-resolution $\boldsymbol{E} \times \boldsymbol{B}$ drift velocity in the LMN coordinate system, calculated from parameters observed by EFW and FGM instruments on Cluster 2 and 3, during the detection of $B_{N}$ signature. Note that these values are calculated assuming that $\boldsymbol{E} \cdot \boldsymbol{B}=0$.

\begin{tabular}{lll}
\hline Observation region & $\begin{array}{l}\text { Cluster 2 } \\
\text { Velocity }\left(\mathrm{km} \mathrm{s}^{-1}\right)\end{array}$ & $\begin{array}{l}\text { Cluster 3 } \\
\text { Velocity }\left(\mathrm{km} \mathrm{s}^{-1}\right)\end{array}$ \\
\hline Before bipolar $B_{N}$ & $(-1,14,-12)$ & $(0,0,-9)$ \\
During & $(-5,-29,-21)$ & $(-8,-67,8)$ \\
After & $(4,21,11)$ & $(0,-4,14)$ \\
\hline
\end{tabular}

magnetosheath was detected by this spacecraft for the whole time interval.

Similar to Cluster 1, the $\boldsymbol{E} \times \boldsymbol{B}$ drift velocities observed by Cluster 2 and 3 before, during and after the passage of structure are presented in Table 2. The variation in the drift velocity represents the motion of plasma surrounding the FTE, before, during and after the passage of the structure.

\section{Discussion}

Bipolar $B_{N}$ signature observations are normally categorized as occurring under the influence of one of two major magnetopause dynamic processes: (i) those signatures without magnetic reconnection, which may be observed due to the passage of a surface wave across the Earth's magnetopause and over the spacecraft location (Sibeck, 1990, 1992; Sibeck and Smith, 1992); and (ii) observations which involve magnetic reconnection and are often categorized as FTEs. For the latter, Fear et al. (2008) suggested that different FTE models can be grouped as one of three types: elbow-shaped bundles of magnetic flux threading through the magnetopause (Russell and Elphic, 1978), a flux rope formed by multiple X-lines (Lee and Fu, 1985) or a flux bulge on the magnetopause driven by time-dependent reconnection at a single X-line (Southwood et al., 1988; Scholer, 1988). Each of these models has certain geometrical characteristics which support specific predictions of their plasma and electromagnetic field properties and which can, in principle, be used to distinguish which model is the most relevant to a given set of observations. We attempt to make this distinction for the structure whose observational characteristics, sampled at high time resolution, we have described above.

Prior to and during the observations of the transient $B_{N}$ signature discussed in this paper, none of the Cluster spacecraft sampled the magnetosheath to measure the magnetic field direction just upstream of the magnetopause. The ACE, Wind and Geotail spacecraft were widely separated in the solar wind, which appears to have led to a lack of consistency in their respective measurements of the IMF orientation (see Sect. 4.1). We note that, when the Cluster spacecraft were located upstream of the bow shock at $\sim 11: 30 \mathrm{UT}$, the IMF data 


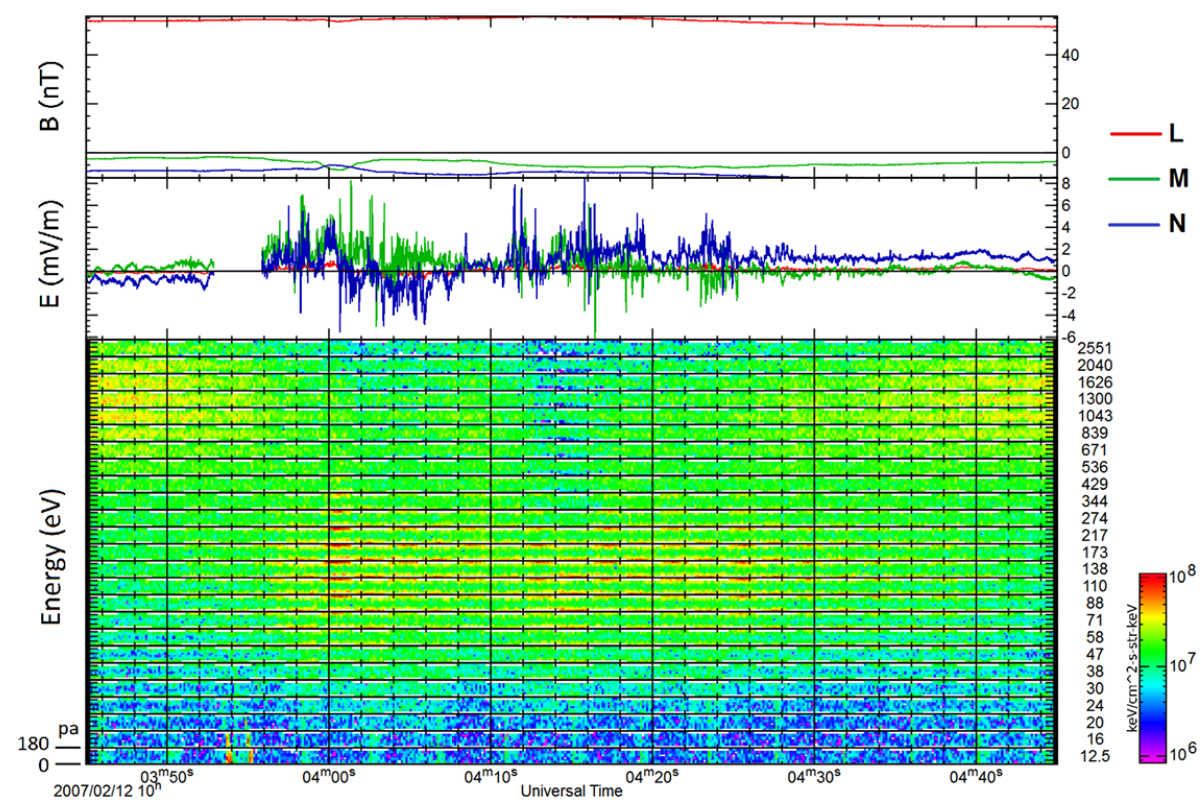

Figure 9. High-time-resolution observations by Cluster 2 from 10:03:45 UT to 10:04:45 UT. Data are shown in the same format as in Fig. 5. Between 10:04:01 UT and 10:04:08 UT, the $B_{N}$ component shows a small bipolar signature, which had an offset $\sim-7 \mathrm{nT}$, due to separation of Cluster 2 from reference spacecraft Cluster 3. The electric field reached a relatively higher level and showed more activity, while the energetic electrons disappeared and a population of bidirectional electrons with energy of $\sim 100 \mathrm{eV}$ were observed. The $B_{\mathrm{L}}$ component reached a slightly higher level throughout the boundary layer indicating that the deflected magnetic field lines were compressed. No entry to magnetosheath is observed for whole time interval. (See Sect. 4.4 .3 for details.)

from Geotail and ACE were in good agreement, after appropriate time lags are applied, with the FGM data from the four Cluster spacecraft. Hence, we assume that the best retrospective estimate of upstream field orientation suggest $\left\langle B_{Z}\right\rangle=0$ during the relevant transient period. Note also that although there is some disparity between measurements of the direction and magnitude of IMF $B_{Z}$, data from all three solar wind spacecraft indicate that a dominant dawnward $B_{Y} \sim-4 \mathrm{nT}$ is likely at the magnetopause between 10:00 and 10:15 UT. The upstream conditions (Sect. 4.1) show that the density, velocity and thus dynamic pressure of the solar wind plasma increased steadily between 10:00 and 13:00 UT on the day of interest, which is expected to have resulted in a continuous inward movement of the magnetopause towards the Earth over this period. This is consistent with the observed timing sequence of the crossings of the four Cluster spacecraft into the magnetosheath. Indeed, our four-spacecraft timing analysis (Sect. 4.3) shows that the magnetopause plane local to the spacecraft was moving Earthwards at a speed of $\sim 44 \mathrm{~km} \mathrm{~s}^{-1}$.

We now mainly focus on the observed transient signatures as they appeared in chronological order. Firstly, Cluster 2, the spacecraft located closest to the noon meridian (Sect. 4.2) observed a bipolar $B_{N}$ between 10:03:45 and 10:04:45 UT, along with electric field noise, an electron population with energy of $\sim 100 \mathrm{eV}$ and dropout of electrons above $6 \mathrm{keV}$. Very similar electromagnetic field and plasma signatures were observed by Cluster 3 between 10:04 and 10:05 UT. At almost the same time, Cluster 4 observed a bipolar $B_{N}$ signature, but the particle measurements showed only the absence of the electrons above $6 \mathrm{keV}$ during that interval. Cluster 1 is the last to observe this signature between 10:05 and 10:06 UT (Sect. 4.4). The sequence and duration of these observations are consistent with a duskward motion of the causative structure, given the relative positions of Cluster quartet, in the LMN coordinate system (Sect. 4.3).

Using the multi-spacecraft timing analysis methods, we estimate the propagation velocity of the structure. Given the observation times at the centre of transient signature (Cluster 2 10:04:18 UT, Cluster 3 10:04:36.5 UT, Cluster 4 10:04:38 UT and Cluster 1 10:05:35 UT), the speed of the structure was $\sim 103 \mathrm{~km} \mathrm{~s}^{-1}$ in the $(-0.039,-0.994,-0.107)$ LMN direction, which indicates that the structure was indeed moving predominantly duskwards across the magnetopause. With this in mind, an approximate azimuthal extent, $\Delta M$, of the structure can be calculated by multiplying the signature durations by the speed, from which we find $1.16 R_{\mathrm{E}} \lesssim \Delta M \lesssim 1.37 R_{\mathrm{E}}$. We note also that Cluster 2 and 3 were separated by $\sim 1.40 R_{\mathrm{E}}$ in the $L$ direction. The close similarity in both the nature and the timing of the signature at these two spacecraft is then consistent with the determination that $V_{L} \sim 0$. Thus the structure clearly is moving along the plane of the magnetopause, and is extended in the $L$ direction. The small variation in the duration of observations 


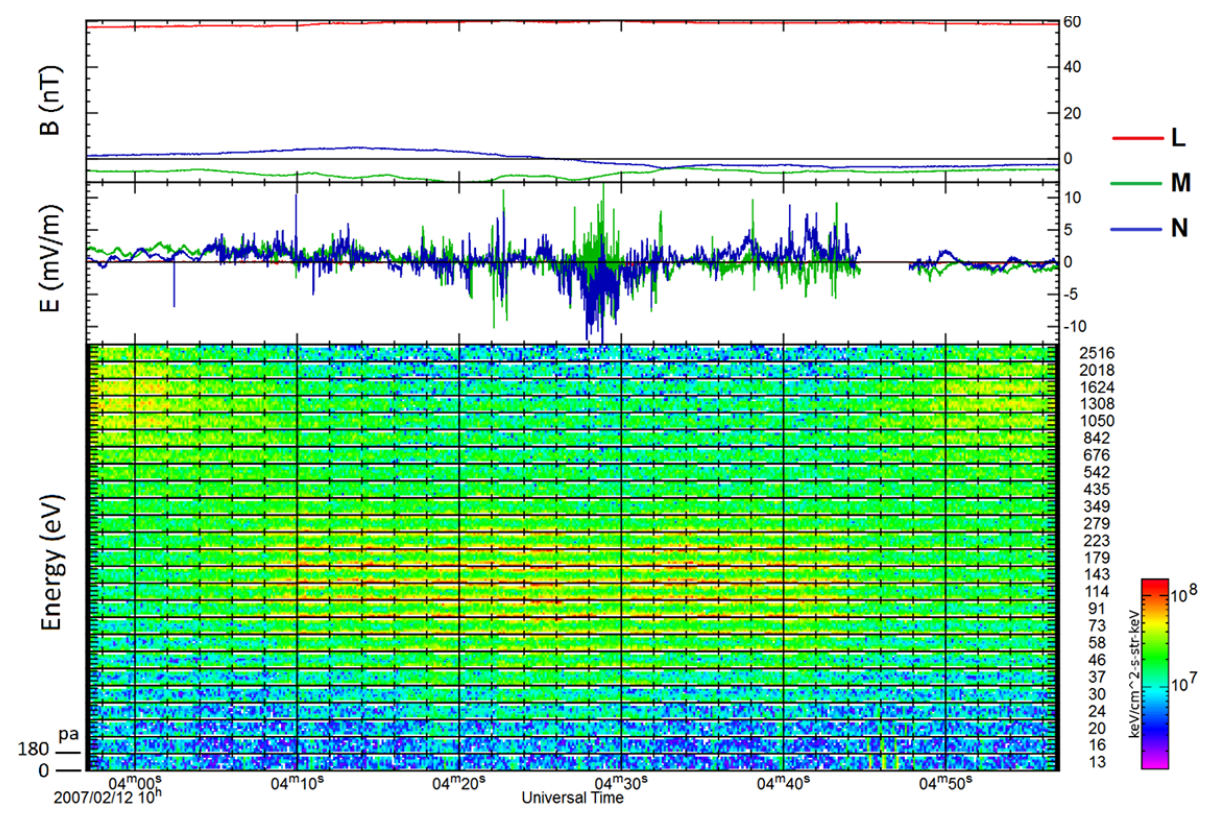

Figure 10. High-time-resolution observations by Cluster 3 from 10:03:57 UT to 10:04:57 UT: data are shown in the same format as in Fig. 5. Between 10:04:14 and 10:04:32 UT, the $B_{N}$ component shows a bipolar signature, while for the electric field and electron pitch angle distribution, similar variations to those observed by Cluster 2 are evident. (See Fig. 9, and Sect. 4.4 .3 for details.)

between spacecraft could be caused by the separation of spacecraft in the $N$ direction. With reference to the location of Cluster 3, which was nominally the closest to the model magnetopause plane at $\sim 10: 04$ UT, Cluster 2 and 4 were respectively $\sim 720$ and $\sim 634 \mathrm{~km}$ further Earthwards. Therefore, the Cluster 3 observations of a longer signature duration in comparison with Cluster 2 and Cluster 4, suggest that the structure is somewhat broader closer to the magnetopause. This is nominally consistent also with the observation by Cluster 1, about one minute later, which was $\sim 272 \mathrm{~km}$ closer to the modelled flat-plane magnetopause than Cluster 3, and observed both the longest duration and clearly passed the deepest into the core of the structure, being the only spacecraft to observe the high fluxes of magnetosheath particles. We note also that the structure might have evolved between the times of observation by $\mathrm{C} 2, \mathrm{C} 3, \mathrm{C} 4$ and its arrival at $\mathrm{C} 1$, resulting in its possible growth. Figure 11, demonstrates the passage of the quartet, and their relative distances.

Given the background of a global inward motion of the magnetopause, it seems unlikely that the bipolar $B_{N}$ signature observed by the four Cluster spacecraft between 10:04 and 10:06 UT is generated by a transient pressure pulse (Sibeck, 1990, 1992; Sibeck and Smith, 1992). Instead there is evidence that the observed signatures are the result of the passage of an FTE. For instance, there is no signature of ongoing periodic wave on the magnetopause, and the timing analysis show that the structure was moving towards the dusk flank. In addition, the plasma signatures are also consistent with the occurrence of transient reconnection; inside the structure seen by $\mathrm{C} 1$ between 10:05 and 10:06 UT, there is a low but measurable flux of electrons in the energy range from 1 to $6 \mathrm{keV}$, in contrast with later observations in the magnetosheath (for example at 10:12 UT) which show that electron fluxes for energies higher than $1 \mathrm{keV}$ were below the PEACE sensitivity. This again indicates a residue of electrons of magnetospheric origin inside the FTE, despite the expectation that these should rapidly evacuate the flux tube once it has become opened. We note also that inside the FTE, the energy of the peak electron flux was $\sim 73 \mathrm{eV}$, while inside the magnetosheath this was $\sim 58 \mathrm{eV}$, suggesting that some process, such as reconnection, had acted to heat and/or accelerate the electrons in the FTE.

The timing analysis, dimensions and motion of this FTE provide some constraints on which models of FTE formation and structure may be relevant. Since the structure was not widely extended in the $M$ direction at this location, it is likely not consistent with the multiple $\mathrm{X}$-line model (Lee and $\mathrm{Fu}$, 1985). Also, the structure moved predominantly duskward. In the single X-line model (Southwood et al., 1988; Scholer, 1988), a transient increase in the reconnection rate causes a bulge to propagate along reconnected field lines. When observed inside the magnetosphere, we would expect the motion of such a bulge to have a significant component in the $L$ direction. Therefore, the best-fitting model based on the propagation of FTE in the $M$ direction, and its extension along the $L$ direction, is perhaps the elbow-shaped-type of FTE (Russell and Elphic, 1978), in which a bundle of reconnected flux tubes are being peeled away from the dayside magnetopause. In other words, given the predominantly dawnward IMF, and spacecraft location in the afternoon 


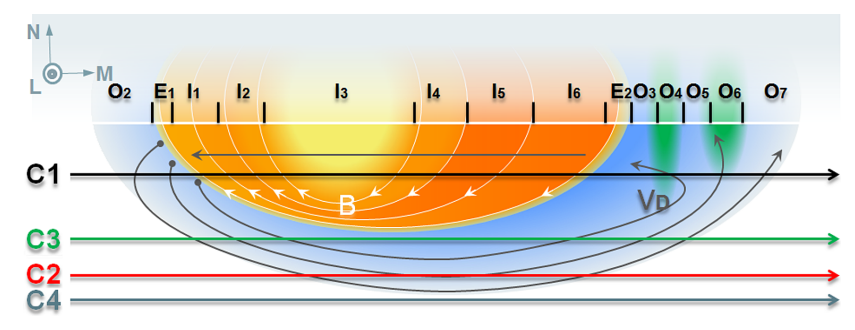

Figure 11. A schematic interpretation of the structure of the FTE and surrounding regions on the $M N$ plane: the relative spacecraft trajectories during the FTE observation are demonstrated in Cluster standard colour code. The four spacecraft do not pass the FTE simultaneously. In fact Cluster 1 was the last spacecraft which observed the FTE, and made the deepest entry. Cluster 3 and 2 only observed the boundary layer, and Cluster 4 only observed the draped magnetospheric field lines. The white arrows represent the projection of the magnetic field onto the $M N$ plane. These magnetic fields also have strong component along the $L$ direction, and separate the multiple plasma layers identified by PEACE. These layers consist of the edges, outer and inner layers of FTE and are marked in black region codes. The colours of these layers illustrate the variation in differential energy flux of the electrons, which are based on LEEA high-time-resolution observations. The grey stream lines (arrowed) are the inferred $\boldsymbol{E} \times \boldsymbol{B}$ drift velocity. These arrows show that the core is moving towards the dusk, while the boundary layer plasmas have a circular motion around the core. In the wake of the FTE, the motion of boundary layer follows the core.

sector, this is consistent with occurrence of a burst of reconnection near the nose of the magnetopause, resulting in a tube of reconnected flux. Using the solar wind observations (Sect. 4.1) and the Cooling et al. (2001) model, the X-line would be required to extend northern dawn to southern dusk. In this structure, the magnetospheric arm would be connected to the northern polar ionosphere, mapping mainly along the $L$ direction at the position of observation, and would be in the process of being dragged duskward across the magnetopause plane as a result of the connection of the magnetosheath arm to the solar wind. This interpretation is also consistent with that made for the event reported by Owen et al. (2008).

We now turn to consider the detailed structure of the FTE and the various plasma regimes observed during its passage over the spacecraft location (Sects. 4.4.2 and 4.4.3). A dropout of the energetic electrons and the appearance of electrons with energies around $100 \mathrm{eV}$ is first observed by Cluster 2 and Cluster 3 in conjunction with the observation of the $B_{N}$ signature at these spacecraft. This is consistent with expectations if Clusters 2 and 3 moved temporarily onto convecting open-field lines (i.e. recently reconnected magnetic field lines in the boundary layer of structure), where the energetic electrons of magnetospheric origin rapidly escaped along the field line after reconnection, and are replaced by a low-energy, higher-density electron population of magnetosheath origin. We note that the latter population mainly consists of bidirectional electrons with energies of $\sim 100 \mathrm{eV}$, which is higher in energy than those observed somewhat later inside the magnetosheath, suggesting that they are accelerated as they cross the magnetopause. Note also that they are observed along with strong electric field fluctuations, with levels of up to $\sim 10-20 \mathrm{mV} \mathrm{m}^{-1}$. Such observations have previously been linked to the encounter of a separatrix layer extending far away from the reconnection diffusion region (André et al., 2004; Owen et al., 2008). Cluster 4, however, observed only a moderate dropout of the energetic electrons associated with the $B_{N}$ signature at that spacecraft, suggesting that this spacecraft may have just grazed the very edge of such a region, since there appears to be some loss of energetic particles without time for the field lines to fill with lowerenergy electrons. Given these observations, we conclude that Cluster 2 and 3 detected the draped closed-field lines around the structure, but they spent significant time in the structure boundary layer, observing open-field lines which have more recently been filled with magnetosheath plasma. Also, Cluster 4, nominally located furthest inside the magnetosphere, observed the bipolar signature in $B_{N}$ but this seems predominantly the result of the draping of closed-field lines over the FTE as it passed by, with perhaps only a brief encounter of very recently opened field lines which have not had time to fill with magnetosheath plasma.

From the high-time-resolution observations of Cluster 1 (see Sect. 4.4.2, Fig. 5), it is evident that C1 was the only spacecraft which observed all three regions identified in previous studies (e.g. Rijnbeek et al., 1987; Farrugia et al., 2011): (1) the draped closed field lines with mainly magnetospheric plasma; (2) deflected exterior open field lines and plasma of the boundary layer; (3) the reconnected magnetic field inside the core of flux tube, with plasma of the magnetosheath. Observations of $\mathrm{C} 1$ (Figs. 5 and 7) in the $\mathrm{O}_{1}$ and $\mathrm{O}_{8}$ regions are consistent with expectations for the plasma population on closed, non- or slowly convecting magnetospheric field lines and are largely unaffected by the presence of the structure. This spacecraft first detected signatures of the approaching FTE at 10:05:04 UT, with the observation of an upstream boundary layer (region $\mathrm{O}_{2}$ ), just before entering the core of flux tube. The magnetic field orientation in this region largely remained at the prior magnetospheric orientation, but the field increased in strength, which we interpret as a signature of the field lines being compressed by either the approaching magnetopause, or the approaching FTE structure itself. The electric field in this region ranged between 10 to $20 \mathrm{mV} \mathrm{m}^{-1}$ with a high level of wave activity. Energetic electrons prevalent in the magnetosphere disappeared, while low fluxes of bidirectional electrons with a peak energy of $114 \mathrm{eV}$ were observed by PEACE. At the same time, the ions are observed to have overall a wider range of energy in this region with a distinct high-energy band ( $\sim 4$ to $\sim 20 \mathrm{keV})$ separated from a lower-energy band (between $\sim 50 \mathrm{eV}$ and $2 \mathrm{keV}$ ). These particle observations are consistent with $\mathrm{C} 1$ encountering a very recently opened set of flux tubes from which the magnetospheric electrons have escaped while the 
magnetosheath electrons are in the process of entering along the magnetic field line. However, these field lines have not been opened long enough for the high-energy ions of magnetospheric origin to have escaped. The region $\mathrm{O}_{2}$ thus may represent a boundary layer with plasma that originates in both the magnetosphere and magnetosheath.

Once the core of the FTE (regions $I_{1}$ to $I_{6}$ ) has passed over the spacecraft, plasma of similar characteristic to region $\mathrm{O}_{2}$ is again observed in the trailing regions marked as $\mathrm{O}_{3}$, $\mathrm{O}_{5}$ and $\mathrm{O}_{7}$. Observations in these regions are also similar to the observations of the boundary layer regions by Cluster 2 and 3, with bidirectionally distributed electrons of energies $\sim 100 \mathrm{eV}$. However, at $\mathrm{C} 1$ these observations are interspersed by observations of plasma in regions $\mathrm{O}_{4}$ and $\mathrm{O}_{6}$ which appear to contain a separate, distinct population only observed by Cluster 1 . The latter group consists of isotropic or nearly isotropic electron populations with energy centred on $58 \mathrm{eV}$, which is close to the average energy observed inside the FTE. A slight reduction in the magnetic field strength, and absence of photoelectric electrons $(\leq 9.5 \mathrm{eV}$ energy), is consistent with these regions $\left(\mathrm{O}_{4}\right.$ and $\left.\mathrm{O}_{6}\right)$ also containing higherdensity plasma than other regions of the boundary layer $\left(\mathrm{O}_{2}\right.$, $\mathrm{O}_{3}, \mathrm{O}_{5}$ and $\mathrm{O}_{7}$ ). In addition to observation of these electron populations, there is also a low-energy, low-density population of ions. However, due to the spin modulation (Fig. 7), more detailed information on the nature of the ions in regions $\mathrm{O}_{4}$ and $\mathrm{O}_{6}$ cannot be obtained as the relevant intervals do not coincide with those in which the CIS instrument is pointing into the flow direction and detecting significant fluxes. We note that there is no evidence of a separate bipolar $B_{N}$ signature to suggest that these intervals may be related to a second distinguishable FTE in those regions, and the electric field activity at similar levels to all the other boundary layer regions. In the wake of the core structure, there is a clear mixture of magnetospheric and magnetosheath plasmas on the same flux tube, which supports our earlier conclusion of FTE being the result of a transient reconnection.

For a better understanding of the motion of plasma in the boundary layer, we have calculated the $\boldsymbol{E} \times \boldsymbol{B}$ drift velocity from the parameters observed by Cluster 1,2 and 3 . For this calculation, it is assumed that $\boldsymbol{E} \cdot \boldsymbol{B}=0$ and therefore errors may exist associated with any possible electric field component parallel to the magnetic field. With this caveat in mind, the drifts in the observed regions are presented in Tables 1 and 2. Just before the arrival of FTE at each spacecraft, the drift velocity measured by $\mathrm{C} 2, \mathrm{C} 3$, and $\mathrm{C} 1$ had negative $V_{N}$, and after the structure passed, has positive $V_{N}$. This is consistent with the magnetic field lines ahead of structure being pushed Earthwards as the FTE was approaching along the magnetopause. The observed compression in the magnetic field at all three spacecraft is also consistent with this interpretation. We note that the magnetopause itself was expected to be affected by solar wind pressure increase (Sect. 4.1), which might have added a total inward motion as well. Within these exterior regions of the FTE however, the $\boldsymbol{E} \times \boldsymbol{B}$ drift at all three spacecraft showed a negative $V_{M}$ component, which indicates the dawnward motion of field lines which are closer to the FTE core. Once the FTE has passed, a positive $V_{N}$ component indicates that the field lines go back towards their initial location by expanding outwards. This positive $V_{N}$ along with positive $V_{M}$ observed by C2 indicates a circulatory motion of field and plasma around the FTE. Such motions were predicted by Farrugia et al. (1987), as they theoretically studied the flow perturbation of plasma past a cylindrical FTE. This hypothesis was then confirmed by observations (e.g. Sibeck and Smith, 1992; Korotova et al., 2009) that FTEs push the magnetospheric plasma in front of them and entrain the behind, and thus a return flow of plasma occurs on the flanks of FTEs and deeper within the magnetosphere. MHD simulations and Grad-Shafranov reconstructions (Birn et al., 2004; Snekvik et al., 2007) show that such motions are also common for other plasma bubbles, such as Bursty Bulk Flows. In situ observations and ionospheric signatures have proven the return flow around these bubbles in the magnetotail (e.g. Kauristie et al., 2000; Pitkänen et al., 2011; Walsh et al., 2009; Walsh and Forsyth, 2011).

Once the structure had passed, Cluster 1 observed the plasma drifting duskwards in the disrupted region in the wake of the FTE. Hence, we propose that, in the wake of the FTE, in the portion of the boundary layer closer to the core, the motion of boundary layer follows the core. One assumption is that these motions might potentially lead to ripples in the wake of the FTE, and result in observation of such isotropic population from magnetosheath origin in regions $\mathrm{O}_{4}$ and $\mathrm{O}_{6}$. Note that the electron moments which are produced at spin resolution $(4.148 \mathrm{~s})$ are too slow to be used to analyse these possible vortices. The summary of these motions are illustrated with grey arrows in Fig. 11, for both inside the core and inside the boundary layer.

Cluster 1 entered the FTE proper at 10:05:08 UT, where the $B_{L}$ component dropped from its maximum level and $B_{N}$ increased from zero to its maximum level in region $\mathrm{E}_{1}$, which we identify as the edge of the structure itself. The electric field strength decreased from its maximum to below $\sim 10 \mathrm{mV} \mathrm{m}^{-1}$ and showed a lower level of activity. At the same time, the electrons of the boundary layer were replaced by higher fluxes of magnetosheath-like electrons. The electromagnetic field variations at the edge of FTEs can be routinely observed by Cluster (e.g. Farrugia et al., 2011), but the benefit of using these high-time-resolution particle distributions is clear during this transition, in which region $\mathrm{E}_{1}$ is crossed in only $0.5 \mathrm{~s}$. At more usual spin resolution, the plasmas from both the boundary layer, the edge and the core would be aliased over $4 \mathrm{~s}$ and thus no clear edge region would be distinguished. The observations in region $\mathrm{E}_{1}$ show that energy flux of electrons increased from $\sim 10^{7}$ to above $\sim 10^{8} \mathrm{keV}\left(\mathrm{cm}^{2} \mathrm{~s} \mathrm{sr} \mathrm{keV}\right)^{-1}$, their typical energy dropped from 114 to $47 \mathrm{eV}$, and their initially bidirectional distribution gradually turned into a more isotropic one, 
broadening first from the field-antiparallel direction and then spreading into a field-parallel beam (e.g. Fig. 6). Note that the reverse sequence (conversion of an isotropic distribution to bidirectional) took place over $\sim 1 \mathrm{~s}$ as the spacecraft crossed the edge region marked $\mathrm{E}_{2}$ as it exited the core interior region. At the boundary between $\mathrm{O}_{2}$ and $\mathrm{E}_{1}$, and also that between $\mathrm{E}_{2}$ and $\mathrm{O}_{3}$, the electric field magnitude reaches the highest values seen during this entire event. This is consistent with the behaviour expected at the two sheets of relatively strong current separating these regions (André et al., 2004, 2010; Vaivads et al., 2004). Given the fact that the average electron energy inside the magnetosheath was $58 \mathrm{eV}$, and inside the boundary layer was $114 \mathrm{eV}$, we highlight the observation of the thin layers of electrons with lower observed temperatures (peak energy: $47 \mathrm{eV}$ ) at the time of the crossing of these current sheets. The existence of cold-accelerated electrons at the edge of FTEs has previously been reported by André et al. (2010), who suggested that these are part of the reconnection current circuit. In addition, the antiparallel beams of electrons observed in these edge regions have higher fluxes than the parallel equivalent just outside the edge of FTE, which may be consistent with a Hall electron current flowing in this region, as previously reported by André et al. (2004) and Vaivads et al. (2004). Minimum variance analysis of $E_{1}$ and $E_{2}$ suggest that the outbound normal vector rotated $\sim 32.5^{\circ}$ dawnwards at the entrance of Cluster into the structure, and then $\sim 30.4^{\circ}$ duskwards at the exit. This again is consistent with a FTE passing by $\mathrm{C} 1$ and moving duskwards.

The PEACE, CIS, EFW and FGM observations recorded within the core of FTE, regions $I_{1}$ to $I_{6}$ (Sect. 4.4.2), show that while the $B_{N}$ component decreased quasi-continuously from its maximum at the start of $\mathrm{I}_{1}$, to its minimum in $\mathrm{I}_{6}$, the $B_{M}$ component was persistently deflected towards dusk and the $B_{L}$ component showed an enhancement at the centre of this interval, in region $\mathrm{I}_{3}$. These observations are consistent with those in previous reports of crater FTEs (e.g. LaBelle et al., 1987). The electric field inside the core region was $<10 \mathrm{mV} \mathrm{m}^{-1}$ throughout and relatively quieter than anywhere else during the interval studied. The average $\boldsymbol{E} \times \boldsymbol{B}$ drift velocity within these interior regions was $\sim 100 \mathrm{~km} \mathrm{~s}^{-1}$ in the $(-0.18,-0.97,-0.16) \mathrm{LMN}$ direction for most of the time, which is in close agreement (within 9 degrees) with the propagation velocity of FTE calculated by multi-spacecraft timing analysis $\left(\sim 103 \mathrm{~km} \mathrm{~s}^{-1}\right.$ in $(-0.039,-0.994,-0.107)$ LMN). From the PEACE observations, we can see a clear temperature change between different regions within the core. Knowing that the minimum temperature of $47 \mathrm{eV}$ occurred at the edges, the electrons are slightly heated in region $I_{1}$ and reach a peak of $58 \mathrm{eV}$ inside $I_{2}$, which is close to the average of electron energy observed later within the magnetosheath. During the same period of time, CIS observations show that the ions moved mainly perpendicular to the concurrently observed magnetic field in the $(-0.19$, $-0.97,-0.17$ ) LMN direction inside each of the $E_{1}, I_{1}$ and
$\mathrm{I}_{2}$ regions, with a steady increase in the energy band containing the peak flux from around 300 to around $500 \mathrm{eV}$.

In region $\mathrm{I}_{3}$, containing a local maximum in the field strength, the electron temperature reduces to the level at the FTE edges. The distribution of electrons remained isotropic in this region. Unfortunately there is no relevant PAD for ions available at this time since the CIS instrument was sampling in the opposite direction to that containing the flow. This cold population of electrons in the central region of the core is observed for the first time and is thus of particular interest. Since the flux and temperature of electrons in this region is similar to those in the edges, it is tempting to relate the observation to a temporary return towards the near-edge region, perhaps due to an oscillatory motion of the magnetopause. In the case of such a return, we would expect to see variations in the $B_{N}$ component with a secondary peak, due to the back and forth displacement of spacecraft relative to the magnetopause boundary. However, the observations show that the $B_{N}$ component decreased from maximum to minimum in a monotonic fashion, while the $B_{M}$ component remained steadily duskwards. These observations are not consistent with a return to the edge. Moreover, the electric field value does not change significantly, and its activity level in this central region is not as high as the edges. Hence we conclude that this is a new, separate region within the core, where lower fluxes of plasma with lower temperature results in a reduced level of diamagnetic depression of the magnetic field in this region.

In $\mathrm{I}_{4}$, the plasma temperature returned to the same level as for $\mathrm{I}_{2}$. Both electrons and ions increased in energy flux, and the ion distribution had components of motion both field aligned and perpendicular to the field. In $\mathrm{I}_{5}$, the plasma reached its highest temperature and also energy flux, suggesting that a heating process continued as the spacecraft crossed the interior regions, but the distribution of particles remained similar to those in $\mathrm{I}_{4}$. Finally, in the last region of the FTE core, $\mathrm{I}_{6}$, highly field-aligned ions are observed along with bidirectional electron fluxes just before exiting the core of FTE. We discuss this latter region in more detail below.

During the passage of Cluster 1 through the core of the FTE, there is spin phase modulation for ions in magnetosheath energies, as they are not gyrotropic in the spacecraft frame (Figs. 7 and 8). Therefore, there are only six data points where near-complete ion pitch angle distributions and unaliased ion moments are reconstructed. We have used these spin resolution moments to calculate the Alfvén speed, which is $\sim 950-1250 \mathrm{~km} \mathrm{~s}^{-1}$ in the boundary layer outside the FTE, and $\sim 95-215 \mathrm{~km} \mathrm{~s}^{-1}$ inside the core. It is to be noted that these are approximate velocities, as there were possibly heavier ions which were not detected by the HIA instrument, and not taken into account in the plasma density. We note that during the last spin inside the FTE, when strong field-aligned ions and bidirectional electrons were observed, the parallel component of ion velocity, $V_{\|} \sim 115 \mathrm{~km} \mathrm{~s}^{-1}$, was comparable to Alfvén velocity at that time. This perhaps might be 
evidence for unwinding kinked reconnected field lines, given the observations made before the rear edge of FTE, where ions were moving along the magnetic field line with speeds comparable to Alfvén speed (Cowley and Owen, 1989; Smith and Owen, 1992; Owen et al., 2001).

Based on these high-time-resolution observations, we postulate that the observed FTE is not an isolated flux tube, but instead the ongoing reconnection on the field lines forming the boundary layer is contributing to the growth of the structure. In other words, while the core of the FTE consists of the initially reconnected field lines forming an FTE, the boundary is also open and is formed of more recently reconnected flux tubes that contain heated plasma, and which are being added to the outer layer of the FTE core. For this particular FTE, clear evidence of the more recent reconnection is seen towards the trailing edge, which will subsequently reach a more stable equilibrium with other regions within the core (Hasegawa et al., 2010).

A summary plot/schematic view of the observations is shown in Fig. 11 on the $M N$ plane. The spacecraft trajectories during the FTE observation are demonstrated in Cluster standard colour code, and based on their distance from magnetopause plane and PEACE observations. The projection of magnetic field onto the $M N$ plane, which was measured by FGM, is shown by white arrows. These magnetic fields also have a strong component along the $L$ direction, which supposedly represents helical field lines, which separate the multiple plasma layers identified by PEACE. These layers consist of the edges, outer and inner layers of FTE and are marked in black letters. The colours of these layers illustrate the variation in differential energy flux of the electrons, which are based on LEEA high-time-resolution observations. The grey arrows are the $\boldsymbol{E} \times \boldsymbol{B}$ drift velocity measured by EFW, both inside and outside the FTE core.

\section{Summary}

In this paper, we have presented the Cluster observations of a crater FTE on 12 February 2007, when the quartet was located in the low-latitude boundary layer, and widely separated on the magnetopause plane. The passage of the FTE was sequentially observed by Cluster 2, 3, 4 and 1 respectively. Cluster 4 observed only the field lines draping around the structure, while Cluster 2 and 3 also observed the deflected open-field lines with bidirectional low-energy electrons. Finally, Cluster 1 observed the core of the FTE in addition to the other regions. Putting the evidence together, including the solar wind conditions, the results of timing analysis, the electromagnetic field variations, the convection speed and direction, and the particle distributions at the four Cluster spacecraft, we conclude that the observations by Cluster are not due to a temporary displacement of the magnetopause over the spacecraft location, as the solar wind dynamic pressure was steadily increasing. We conclude that these observations are consistent with the encounter of the magnetospheric portion of a crater-type FTE consisting of a north-south (magnetospheric field) aligned tube of reconnected flux, as expected from the elbow-shaped model, convecting in the duskward direction. Given the predominantly dawnward IMF, this is consistent with the pulling of the magnetospheric arm of the flux rope duskward across the magnetopause plane under the influence of magnetic tension. However, since the magnetosheath counterpart of this FTE signature is not observed by any of the spacecraft, and due to IMF conditions, the topology of the reconnection region might be much more complicated than the one originally sketched by Russell and Elphic (1978). During the event of primary interest, all four spacecraft were operating in burst mode, and the magnetic field orientation inside the magnetosphere was nearly along the spin axis of the spacecraft; thus, we have used the 3-D data from PEACE and CIS instruments to reconstruct the high-time-resolution pitch angle distribution of electrons and ions. In this way, we have thus used the maximum capability of Cluster to study the plasma with up to 32 times higher time resolution than is normally available. As a result, we have been able to identify an FTE substructure consisting of a number of different layers. The main results of the analysis in this paper are as follows:

1. Identification of multiple layers of plasma, and a general heating of the plasma populations towards the edges of the FTE core.

2. Identification of a central region during the magnetic field enhancement period, with the lowest flux and lowest temperature plasma, compared to other regions of the core of the FTE.

3. Identification of strongly field-aligned ions with Alfvénic speeds along with bidirectional electrons near the rearward edge of the FTE.

4. Identification of thin current layers at the FTE edges, where the bidirectional distribution of electrons gradually turned into a more isotropic one, broadening first from the antiparallel direction and then spreading into the parallel beam.

5. Identification of isotropically distributed electrons in the FTE boundary layer.

Further studies of similar events will contribute to understanding the processes involved in layering the plasma within the FTE. We believe that these observations are relevant precursors which may be useful in preparation for higher time resolution missions in the future, such as NASA's Magnetospheric Multiscale (MMS) mission. 
Acknowledgements. We thank the Cluster instrument teams and the Cluster Active Archive for providing the data used in this study. C. J. Owen, A. N. Fazakerley and C. Forsyth were supported by STFC Consolidated Grant ST/K000977/1. A. P. Walsh was supported by an ESA Postdoctoral Fellowship. Certain plots and also analysis in the paper has been performed using the QSAS science analysis software for space plasma data.

Topical Editor E. Roussos thanks two anonymous referees for their help in evaluating this paper.

\section{References}

André, M., Vaivads, A., Buchert, S. C., Fazakerley, A. N., and Lahiff, A.: Thin electron-scale layers at the magnetopause, Geophys. Res. Lett., 31, L03803, doi:10.1029/2003g1018137, 2004.

André, M., Vaivads, A., Khotyaintsev, Y. V., Laitinen, T., Nilsson, H., Stenberg, G., Fazakerley, A., and Trotignon, J. G.: Magnetic reconnection and cold plasma at the magnetopause, Geophys. Res. Lett., 37, L22108, doi:10.1029/2010g1044611, 2010.

Balogh, A., Carr, C. M., Acuña, M. H., Dunlop, M. W., Beek, T. J., Brown, P., Fornacon, K.-H., Georgescu, E., Glassmeier, K.H., Harris, J., Musmann, G., Oddy, T., and Schwingenschuh, K.: The Cluster Magnetic Field Investigation: overview of in-flight performance and initial results, Ann. Geophys., 19, 1207-1217, doi:10.5194/angeo-19-1207-2001, 2001.

Berchem, J. and Russell, C. T.: Flux transfer events on the magnetopause: Spatial distribution and controlling factors, J. Geophys. Res.-Space, 89, 6689-6703, doi:10.1029/JA089iA08p06689, 1984.

Birn, J., Raeder, J., Wang, Y. L., Wolf, R. A., and Hesse, M.: On the propagation of bubbles in the geomagnetic tail, Ann. Geophys., 22, 1773-1786, doi:10.5194/angeo-22-1773-2004, 2004.

Cooling, B. M. A., Owen C. J., and Schwartz, S. J.: Role of the magnetosheath flow in determining the motion of open flux tubes, J. Geophys. Res.-Space, 106, 18763-18776, doi:10.1029/2000JA000455, 2001.

Cowley, S. W. H.: The causes of convection in the Earth's magnetosphere: A review of developments during the IMS, Rev. Geophys., 20, 531-565, doi:10.1029/RG020i003p00531, 1982.

Cowley, S. W. H. and Owen, C. J.: A simple illustrative model of open flux tube motion over the dayside magnetopause, Planet. Space Sci., 37, 1461-1475, doi:10.1016/0032-0633(89)90116-5, 1989.

Daly, P. W., Saunders, M. A., Rijnbeek, R. P., Sckopke, N., and Russell, C. T.: The distribution of reconnection geometry in flux transfer events using energetic ion, plasma and magnetic data, J. Geophys. Res.-Space, 89, 3843-3854, doi:10.1029/JA089iA06p03843, 1984.

Dungey, J. W.: Interplanetary magnetic field and auroral zones, Phys. Rev. Lett., 6, 47-48, doi:10.1103/PhysRevLett.6.47, 1961.

Dunlop, M. W., Taylor, M. G. G. T., Davies, J. A., Owen, C. J., Pitout, F., Fazakerley, A. N., Pu, Z., Laakso, H., Bogdanova, Y. V., Zong, Q.-G., Shen, C., Nykyri, K., Lavraud, B., Milan, S. E., Phan, T. D., Rème, H., Escoubet, C. P., Carr, C. M., Cargill, P., Lockwood, M., and Sonnerup, B.: Coordinated Cluster/Double Star observations of dayside reconnection signatures, Ann. Geophys., 23, 2867-2875, doi:10.5194/angeo-23-2867-2005, 2005.

Elphic, R. C., Baumjohann, W., Cattell, C. A., Lühr, H., and Smith, M. F.: A search for upstream pressure pulses associated with flux transfer events: An AMPTE/ISEE case study, J. Geophys. Res.Space, 99, 13521-13527, doi:10.1029/94ja00575, 1994.

Escoubet, C. P., Fehringer, M., and Goldstein, M.: Introduction The Cluster mission, Ann. Geophys., 19, 1197-1200, doi:10.5194/angeo-19-1197-2001, 2001.

Farrugia, C. J., Elphic, R. C., Southwood, D. J., and Cowley, S. W. H.: Field and flow perturbations outside the reconnected field line region in flux transfer events: Theory, Planet. Space Sci., 35, 227-240, 1987.

Farrugia, C. J., Rijnbeek, R. P., Saunders, M. A., Southwood, D. J., Rodgers, D. J., Smith, M. F., Chaloner, C. P., Hall, D. S., Christiansen, P. J., and Woolliscroft, L. J. C.: A multi-instrument study of flux transfer event structure, J. Geophys. Res.-Space, 93, 14465-14477, doi:10.1029/JA093iA12p14465, 1988.

Farrugia, C. J., Chen, L., Torbert, R. B., Southwood, D. J., Cowley, S. W. H., Vrublevskis, A., Mouikis, C., Vaivads, A., André, M., Décréau, P., Vaith, H., Owen, C. J., Sibeck, D. J., Lucek, E., and Smith, C. W.: "Crater" flux transfer events: Highroad to the X line?, J. Geophys. Res.-Space, 116, A02204, doi:10.1029/2010ja015495, 2011.

Fazakerley, A. N., Lahiff, A. D., Wilson, R. J., Rozum, I., Anekallu, C., West, M., and Bacai, H.: PEACE Data in the Cluster Active Archive, in: The Cluster Active Archive, Studying the Earth's Space Plasma Environment, edited by: Laakso, H., Taylor, M. G. T. T., and Escoubet, C. P., Astrophysics and Space Science Proceedings, Berlin: Springer, 129-144, doi:10.1007/978-90-4813499-1_8, 2010.

Fear, R. C., Fazakerley, A. N., Owen, C. J., and Lucek, E. A.: A survey of flux transfer events observed by Cluster during strongly northward IMF, Geophys. Res. Lett., 32, L18105, doi:10.1029/2005g1023811, 2005.

Fear, R. C., Milan, S. E., Fazakerley, A. N., Lucek, E. A., Cowley, S. W. H., and Dandouras, I.: The azimuthal extent of three flux transfer events, Ann. Geophys., 26, 2353-2369, doi:10.5194/angeo-26-2353-2008, 2008.

Fear, R. C., Milan, S. E., Fazakerley, A. N., Fornaçon, K. H., Carr, C. M., and Dandouras, I.: Simultaneous observations of flux transfer events by THEMIS, Cluster, Double Star, and SuperDARN: Acceleration of FTEs, J. Geophys. Res.-Space, 114, A10213, doi:10.1029/2009ja014310, 2009.

Fear, R. C., Milan, S. E., Lucek, E. A., Cowley, S. W. H., and Fazakerley, A. N.: Mixed azimuthal scales of flux transfer events, in: The Cluster Active Archive, Studying the Earth's Space Plasma Environment, edited by: Laakso, H., Taylor, M. G. T. T., and Escoubet, C. P., Astrophysics and Space Science Proceedings, Berlin: Springer, 389-398, doi:10.1007/978-90-481-3499-1_27, 2010.

Fear, R. C., Milan, S. E., and Oksavik, K.: Determining the axial direction of high-shear flux transfer events: Implications for models of FTE structure, J. Geophys. Res.-Space, 117, A09220, doi:10.1029/2012ja017831, 2012.

Goertz, C. K., Nielsen, E., Korth, A., Glassmeier, K. H., Haldoupis, C., Hoeg, P., and Hayward, D.: Observations of a possible ground signature of flux transfer events, J. Geophys. Res.-Space, 90, 4069-4078, doi:10.1029/JA090iA05p04069, 1985.

Gustafsson, G., Boström, R., Holback, B., Holmgren, G., Lundgren, A., Stasiewicz, K., Åhlén, L., Mozer, F. S., Pankow, D., Harvey, P., Berg, P., Ulrich, R., Pedersen, A., Schmidt, R., Butler, A., Fransen, A. W. C., Klinge, D., Thomsen, M., Fälthammar, C. G., 
Lindqvist, P. A., Christenson, S., Holtet, J., Lybekk, B., Sten, T. A., Tanskanen, P., Lappalainen, K., and Wygant, J.: The electric field and wave experiment for the Cluster mission, Space Sci. Rev., 79, 137-156, doi:10.1023/a:1004975108657, 1997.

Harvey, C. C.: Spatial Gradients and the Volumetric Tensor, Analysis Methods for Multi-Spacecraft Data, edited by: Paschmann, G. and Daly, P. W., 307-322, ISSI Scientific Report, SR-001, 1998.

Hasegawa, H., Sonnerup, B. U. Ö., Owen, C. J., Klecker, B., Paschmann, G., Balogh, A., and Rème, H.: The structure of flux transfer events recovered from Cluster data, Ann. Geophys., 24, 603-618, doi:10.5194/angeo-24-603-2006, 2006.

Hasegawa, H., Wang, J., Dunlop, M. W., Pu, Z. Y., Zhang, Q. H., Lavraud, B., Taylor, M., Constantinescu, O. D., Berchem, J., Angelopoulos, V., McFadden, J. P., Frey, H. U., Panov, E. V., Volwerk, M., and Bogdanova, Y. V.: Evidence for a flux transfer event generated by multiple $\mathrm{X}$-line reconnection at the magnetopause, Geophys. Res. Lett., 37, L16101, doi:10.1029/2010g1044219, 2010 .

Johnstone, A. D., Alsop, C., Burge, S., Carter, P. J., Coates, A. J., Coker, A. J., Fazakerley, A. N., Grande, M., Gowen, R. A., Gurgiolo, C., Hancock, B. K., Narheim, B., Preece, A., Sheather, P. H., Winningham, J. D., and Woodliffe, R. D.: Peace: A plasma electron and current experiment, Space Sci. Rev., 79, 351-398, doi:10.1023/a:1004938001388, 1997.

Kauristie, K., Sergeev, V. A., Kubyshkina, M., Pulkkinen, T. I., Angelopoulos, V., Phan, T., Lin, R. P., and Slavin J. A.: Ionospheric current signatures of transient plasma sheet flows, J. Geophys. Res., 105, 10677-10690, doi:10.1029/1999JA900487, 2000.

Kawano, H. and Russell, C. T.: Survey of flux transfer events observed with the ISEE 1 spacecraft: Dependence on the interplanetary magnetic field, J. Geophys. Res.-Space, 102, 11307-11313, doi:10.1029/97ja00481, 1997.

Khotyaintsev, Y. V., Vaivads, A., Retinò, A., and André, M.: Formation of inner structure of a reconnection separatrix region, Phys. Rev. Lett., 97, 205003, doi:10.1103/PhysRevLett.97.205003, 2006.

Klumpar, D. M., Fuselier, S. A., and Shelley, E. G.: Ion composition measurements within magnetospheric flux transfer events, Geophys. Res. Lett., 17, 2305-2308, doi:10.1029/GL017i013p02305, 1990.

Kokubun, S., Yamamoto, T., Acuña, M. H., Hayashi, K., Shiokawa, K., and Kawano, H.: The geotail magnetic field experiment, J. Geomagn. Geoelectr., 46, 7-21, doi:10.5636/jgg.46.7, 1994.

Korotova, G. I., Sibeck, D. G., and Rosenberg, T.: Geotail observations of FTE velocities, Ann. Geophys., 27, 83-92, doi:10.5194/angeo-27-83-2009, 2009.

LaBelle, J., Treumann, R. A., Haerendel, G., Bauer, O. H., Paschmann, G., Baumjohann, W., Lühr, H., Anderson, R. R., Koons, H. C., and Holzworth, R. H.: AMPTE IRM observations of waves associated with flux transfer events in the magnetosphere, J. Geophys. Res.-Space, 92, 5827-5843, doi:10.1029/JA092iA06p05827, 1987.

Lee, L. C. and Fu, Z. F.: A theory of magnetic flux transfer at the earth's magnetopause, Geophys. Res. Lett., 12, 105-108, doi:10.1029/GL012i002p00105, 1985.

Lepping, R. P., Acũna, M. H., Burlaga, L. F., Farrell, W. M., Slavin, J. A., Schatten, K. H., Mariani, F., Ness, N. F., Neubauer, F. M., Whang, Y. C., Byrnes, J. B., Kennon, R. S., Panetta, P. V., Scheifele, J., and Worley E. M.: The WIND magnetic field in- vestigation, The Global Geospace Mission, Space Sci. Rev. 71, 207-229, 1995.

Lockwood, M.: Flux-transfer events at the dayside magnetopause - transient reconnection or magnetosheath dynamic pressure pulses?, J. Geophys. Res.-Space, 96, 5497-5509, doi:10.1029/90ja02389, 1991.

Lockwood, M., Cowley, S. W. H., Sandholt, P. E., and Lepping, R. P.: The ionospheric signatures of flux transfer events and solar-wind dynamic pressure changes, J. Geophys. Re.-Space, 95, 17113-17135, doi:10.1029/JA095iA10p17113, 1990.

Mozer, F. S., Bale, S. D., McFadden, J. P., and Torbert, R. B.: New features of electron diffusion regions observed at subsolar magnetic field reconnection sites, Geophys. Res. Lett., 32, L24102, doi:10.1029/2005g1024092, 2005.

Mukai, T., Machida, S., Saito, Y., Hirahara, M., Terasawa, T., Kaya, N., Obara, T., Ejiri, M., and Nishida, A.: The low energy particle (LEP) experiment onboard the Geotail satellite, J. Geomagn. Geoelectr., 46, 669-692, 1994.

Øieroset, M., Lühr, H., Moen, J., Moretto, T., and Sandholt, P. E.: Dynamical auroral morphology in relation to ionospheric plasma convection and geomagnetic activity: Signatures of magnetopause $\mathrm{X}$ line dynamics and flux transfer events, J. Geophys. Res.-Space, 101, 13275-13292, doi:10.1029/96ja00613, 1996.

Ogilvie, K. W., Chornay, D. J., Fritzenreiter, R. J., Hunsaker, F., Keller, J., Lobell, J., Miller, G., Scudder, J. D., Sittler Jr., E. C., Torbert, R. B., Bodet, D., Needell, G., Lazarus, A. J., Steinberg, J. T., Tappan, J. H., Mavretic, A., and Gergin, E.: SWE, A comprehensive plasma instrument for the WIND spacecraft, The Global Geospace Mission, Space Sci. Rev., 71, 55-77, 1995.

Owen, C. J. and Cowley, S. W. H.: Simple models of timedependent reconnection in a collision-free plasma with an application to substorms in the geomagnetic tail, Planet. Space Sci., 35, 451-466, doi:10.1016/0032-0633(87)90102-4, 1987.

Owen, C. J., Fazakerley, A. N., Carter, P. J., Coates, A. J., Krauklis, I. C., Szita, S., Taylor, M. G. G. T., Travnicek, P., Watson, G., Wilson, R. J., Balogh, A., and Dunlop, M. W.: Cluster PEACE observations of electrons during magnetospheric flux transfer events, Ann. Geophys., 19, 1509-1522, doi:10.5194/angeo-191509-2001, 2001.

Owen, C. J., Marchaudon, A., Dunlop, M. W., Fazakerley, A. N., Bosqued, J. M., Dewhurst, J. P., Fear, R. C., Fuselier, S. A., Balogh, A., and Rème, H.: Cluster observations of "crater" flux transfer events at the dayside high-latitude magnetopause, J. Geophys. Res.-Space, 113, A07S04, doi:10.1029/2007ja012701, 2008.

Paschmann, G., Sonnerup, B. U. Ö., Papamastorakis, I., Sckopke, N., Haerendel, G., Bame, S. J., Asbridge, J. R., Gosling, J. T., Russell, C. T., and Elphic, R. C.: Plasma acceleration at the Earth's magnetopause - evidence for reconnection, Nature, 282, 243-246, doi:10.1038/282243a0, 1979.

Paschmann, G., Haerendel, G., Papamastorakis, I., Sckopke, N., Bame, S. J., Gosling, J. T., and Russell, C. T.: Plasma and magnetic-field characteristics of magnetic-flux transfer events, J. Geophys. Res.-Space, 87, 2159-2168, doi:10.1029/JA087iA04p02159, 1982.

Pitkänen, T., Aikio, A. T., Amm, O., Kauristie, K., Nilsson, H., and Kaila, K. U.: EISCAT-Cluster observations of quiet-time near-Earth magnetotail fast flows and their signatures in the 
ionosphere, Ann. Geophys., 29, 299-319, doi:10.5194/angeo-29299-2011, 2011.

Rème, H., Aoustin, C., Bosqued, J. M., Dandouras, I., Lavraud, B., Sauvaud, J. A., Barthe, A., Bouyssou, J., Camus, Th., Coeur-Joly, O., Cros, A., Cuvilo, J., Ducay, F., Garbarowitz, Y., Medale, J. L., Penou, E., Perrier, H., Romefort, D., Rouzaud, J., Vallat, C., Alcaydé, D., Jacquey, C., Mazelle, C., d'Uston, C., Möbius, E., Kistler, L. M., Crocker, K., Granoff, M., Mouikis, C., Popecki, M., Vosbury, M., Klecker, B., Hovestadt, D., Kucharek, H., Kuenneth, E., Paschmann, G., Scholer, M., Sckopke, N., Seidenschwang, E., Carlson, C. W., Curtis, D. W., Ingraham, C., Lin, R. P., McFadden, J. P., Parks, G. K., Phan, T., Formisano, V., Amata, E., Bavassano-Cattaneo, M. B., Baldetti, P., Bruno, R., Chionchio, G., Di Lellis, A., Marcucci, M. F., Pallocchia, G., Korth, A., Daly, P. W., Graeve, B., Rosenbauer, H., Vasyliunas, V., McCarthy, M., Wilber, M., Eliasson, L., Lundin, R., Olsen, S., Shelley, E. G., Fuselier, S., Ghielmetti, A. G., Lennartsson, W., Escoubet, C. P., Balsiger, H., Friedel, R., Cao, J.-B., Kovrazhkin, R. A., Papamastorakis, I., Pellat, R., Scudder, J., and Sonnerup, B.: First multispacecraft ion measurements in and near the Earth's magnetosphere with the identical Cluster ion spectrometry (CIS) experiment, Ann. Geophys., 19, 1303-1354, doi:10.5194/angeo19-1303-2001, 2001.

Retinò, A., Vaivads, A., André, M., Sahraoui, F., Khotyaintsev, Y., Pickett, J. S., Cattaneo, M. B. B., Marcucci, M. F., Morooka, M., Owen, C. J., Buchert, S. C., and Cornilleau-Wehrlin, N.: Structure of the separatrix region close to a magnetic reconnection X-line: Cluster observations, Geophys. Res. Lett., 33, L06101, doi:10.1029/2005g1024650, 2006.

Rijnbeek, R. P., Cowley, S. W. H., Southwood, D. J., and Russell, C. T.: Observations of reverse polarity flux transfer events at the Earth's dayside magnetopause, Nature, 300, 23-26, doi:10.1038/300023a0, 1982.

Rijnbeek, R. P., Cowley, S. W. H., Southwood, D. J., and Russell, C. T.: A survey of dayside flux transfer events observed by ISEE 1 and 2 magnetometers, J. Geophys. Res.-Space, 89, 786-800, doi:10.1029/JA089iA02p00786, 1984.

Rijnbeek, R. P., Farrugia, C. J., Southwood, D. J., Dunlop, M. W., Mierjedrzejowicz, W. A. C., Chaloner, C. P., Hall, D. S., and Smith, M. F.: A magnetic boundary signature within flux transfer events, Planet. Space Sci., 35, 871-878, doi:10.1016/00320633(87)90065-1, 1987.

Rodger, A. S. and Pinnock, M.: The ionospheric response to flux transfer events: the first few minutes, Ann. Geophys., 15, 685691, doi:10.1007/s00585-997-0685-y, 1997.

Roelof, E. C. and Sibeck, D. G.: Magnetopause shape as a bivariate function of interplanetary magnetic field $B_{z}$ and solar wind dynamic pressure, J. Geophys. Res.-Space, 98, 21421-21450, doi:10.1029/93ja02362, 1993.

Roelof, E. C. and Sibeck, D. G.: Correction to "Magnetopause shape as a bivariate function of interplanetary magnetic field $B_{z}$ and solar wind dynamic pressure", J. Geophys. Res.-Space, 99, 8787-8788, doi:10.1029/94ja00348, 1994.

Russell, C. T. and Elphic, R. C.: Initial ISEE magnetometer results - Magnetopause observations, Space Sci. Rev., 22, 681715, doi:10.1007/BF00212619, 1978.

Russell, C. T., Mellott, M. M., Smith, E. J., and King, J. H.: Multiple spacecraft observations of interplanetary shocks: Four space- craft determination of shock normals, J. Geophys. Res.-Space, 88, 4739-4748, doi:10.1029/JA088iA06p04739, 1983.

Saunders, M. A., Russell, C. T., and Sckopke, N.: Flux transfer events: Scale size and interior structure, Geophys. Res. Lett., 11, 131-134, doi:10.1029/GL011i002p00131, 1984.

Scholer, M.: Magnetic flux transfer at the magnetopause based on single X line bursty reconnection, Geophys. Res. Lett., 15, 291294, doi:10.1029/GL015i004p00291, 1988.

Scudder, J. D., Holdaway, R. D., Glassberg, R., and Rodriguez, S. L.: Electron diffusion region and thermal demagnetization, J. Geophys. Res.-Space Phys., 113, A10208, doi:10.1029/2008ja013361, 2008.

Scudder, J. D., Holdaway, R. D., Daughton, W. S., Karimabadi, H., Roytershteyn, V., Russell, C. T., and Lopez, J. Y.: First Resolved Observations of the Demagnetized Electron-Diffusion Region of an Astrophysical Magnetic-Reconnection Site, Phys. Rev. Lett., 108, 225005, doi:10.1103/PhysRevLett.108.225005, 2012.

Shue, J. H., Chao, J. K., Fu, H. C., Russell, C. T., Song, P., Khurana, K. K., and Singer, H. J.: A new functional form to study the solar wind control of the magnetopause size and shape, J. Geophys. Res.-Space, 102, 9497-9511, doi:10.1029/97ja00196, 1997.

Schwartz, S. J., Daly, P. W., and Fazakerley, A. N.: Multi-Spacecraft Analysis of Plasma Kinetics, in: Analysis Methods for MultiSpacecraft Data, edited by: Paschmann, G. and Daly, P. W., 159184, ISSI Scientific Report, SR-001, 1998.

Schwartz, S. J., Henley, E., Mitchell, J., and Krasnoselskikh, V.: Electron Temperature Gradient Scale at Collisionless Shocks, Phys. Rev. Lett., 107, 215002, doi:10.1103/PhysRevLett.107.215002, 2011.

Sibeck, D. G.: A model for the transient magnetospheric response to sudden solar-wind dynamic pressure variations, J. Geophys. Res.-Space, 95, 3755-3771, doi:10.1029/JA095iA04p03755, 1990.

Sibeck, D. G.: Transient events in the outer magnetosphere - boundary waves or flux transfer events?, J. Geophys. Res.-Space, 97, 4009-4026, doi:10.1029/91ja03017, 1992.

Sibeck, D. G. and Siscoe, G. L.: Downstream properties of magnetic flux transfer events, J. Geophys. Res.-Space, 89, 10709-10715, doi:10.1029/JA089iA12p10709, 1984.

Sibeck, D. G. and Smith, M. F.: Magnetospheric plasma flows associated with boundary waves and flux transfer events, Geophys. Res. Lett., 19, 1903-1906, doi:10.1029/92gl01614, 1992.

Smith, M. F. and Owen, C. J.: Temperature anisotropies in a magnetospheric FTE, Geophys. Res. Lett., 19, 1907-1910, doi:10.1029/92g101618, 1992.

Snekvik, K., Haaland, S., Østgaard, N., Hasegawa, H., Nakamura, R., Takada, T., Juusola, L., Amm, O., Pitout, F., Rème, H., Klecker, B., and Lucek, E. A.: Cluster observations of a field aligned current at the dawn flank of a bursty bulk flow, Ann. Geophys., 25, 1405-1415, doi:10.5194/angeo-25-1405-2007, 2007.

Song, P., Le, G., and Russell, C. T.: Observational differences between flux transfer events and surface waves at the magnetopause, J. Geophys. Res.-Space, 99, 2309-2320, doi:10.1029/93ja02852, 1994.

Sonnerup, B. U. Ö. and Cahill, L. J.: Magnetopause structure and attitude from Explorer 12 observations, J. Geophys. Res., 72, 171183, doi:10.1029/JZ072i001p00171, 1967. 
Sonnerup, B. U. Ö. and Scheible, M.: Minimum and maximum variance analysis, in: Analysis Methods for Multi-Spacecraft Data, edited by: Paschmann, G. and Daly, P. W., 185-220, ISSI, 1998.

Sonnerup, B. U. Ö., Paschmann, G., Papamastorakis, I., Sckopke, N., Haerendel, G., Bame, S. J., Asbridge, J. R., Gosling, J. T., and Russell, C. T.: Evidence for magnetic-field reconnection at the Earth's magnetopause, J. Geophys. Res.-Space, 86, 49-67, doi:10.1029/JA086iA12p10049, 1981.

Sonnerup, B. U. Ö., Hasegawa, H., and Paschmann, G.: Anatomy of a flux transfer event seen by Cluster, Geophys. Res. Lett., 31, L11803, doi:10.1029/2004g1020134, 2004.

Southwood, D. J.: The ionospheric signature of flux transfer events, J. Geophys. Res.-Space, 92, 3207-3213, doi:10.1029/JA092iA04p03207, 1987.

Southwood, D. J., Farrugia, C. J., and Saunders, M. A.: What are flux transfer events?, Planet. Space Sci., 36, 503-508, doi:10.1016/0032-0633(88)90109-2, 1988.

Stone, E. C., Frandsen, A. M., Mewaldt, R. A., Christian, E. R., Margolies, D., Ormes, J. F., and Snow, F.: The Advanced Composition Explorer, Space Sci. Rev., 86, 1-22, doi:10.1023/a:1005082526237, 1998.

Thomsen, M. F., Stansberry, J. A., Bame, S. J., Fuselier, S. A., and Gosling, J. T.: Ion and electron velocity distributions within flux transfer events, J. Geophys. Res.-Space, 92, 12127-12136, doi:10.1029/JA092iA11p12127, 1987.
Vaivads, A., Khotyaintsev, Y., André, M., Retinò, A., Buchert, S. C., Rogers, B. N., Décréau, P., Paschmann, G., and Phan, T. D.: Structure of the magnetic reconnection diffusion region from four-spacecraft observations, Phys. Rev. Lett., 93, 105001, doi:10.1103/PhysRevLett.93.105001, 2004.

Walsh, A. P. and Forsyth, C.: Cluster observations of plasma bubbles, BBFs and their wakes, in: The Dynamic magnetosphere, Springer-Verlag Berlin, Berlin, 117-124, doi:10.1007/978-94007-0501-2_6, 2011.

Walsh, A. P., Fazakerley, A. N., Lahiff, A. D., Volwerk, M., Grocott, A., Dunlop, M. W., Lui, A. T. Y., Kistler, L. M., Lester, M., Mouikis, C., Pu, Z., Shen, C., Shi, J., Taylor, M. G. G. T., Lucek, E., Zhang, T. L., and Dandouras, I.: Cluster and Double Star multipoint observations of a plasma bubble, Ann. Geophys., 27, 725-743, doi:10.5194/angeo-27-725-2009, 2009.

Wild, J. A., Cowley, S. W. H., Davies, J. A., Khan, H., Lester, M., Milan, S. E., Provan, G., Yeoman, T. K., Balogh, A., Dunlop, M. W., Fornaçon, K.-H., and Georgescu, E.: First simultaneous observations of flux transfer events at the high-latitude magnetopause by the Cluster spacecraft and pulsed radar signatures in the conjugate ionosphere by the CUTLASS and EISCAT radars, Ann. Geophys., 19, 1491-1508, doi:10.5194/angeo-191491-2001, 2001. 